\title{
Combining Modeling and Measurements to Predict Crystal Morphology in Material Extrusion
}

\author{
Claire McIlroy, ${ }^{1, *}$ Jonathan E. Seppala, ${ }^{2}$ and Anthony P. Kotula ${ }^{2}$ \\ ${ }^{1}$ School of Mathematical Sciences, University of Nottingham, Nottingham, \\ England, NG7 2QL, United Kingdom \\ ${ }^{2}$ Materials Science and Engineering Division, National Institute for \\ Standards and Technology, Gaithersburg, MD 20899, United States \\ "E-mail: claire.mcilroy@nottingham.ac.uk
}

\begin{abstract}
Semicrystalline polymer melts are commonly used in material extrusion (MatEx) for 3D printing. Although flows have a profound effect on polymer crystallization, the relationship between typical MatEx deformation rates and printed-part crystal morphology is yet to be understood. Here, MatEx is used to print a wall of polylactic acid filaments. The linear rheology and quiescent crystallization kinetics are characterized, infrared imaging is used to measure temperature variations during the MatEx process, and optical microscopy is employed to determine the resulting crystal morphology before and after a postprinting thermal annealing process. Our flow-enhanced crystallization model demonstrates that MatEx-induced polymer stretch leads to a higher nucleation density and greater space filling in the weld regions between deposited filaments. Consequently, after annealing, the weld regions feature smaller spherulites than the filament center, as shown by optical microscopy. Finally, flow-induced crystallization is proposed as a method to improve weld toughness.
\end{abstract}




\section{Introduction}

Material extrusion (MatEx) remains the cheapest additive manufacturing technique for printing high-performance thermoplastics, offering advantages such as mass customization, locally controlled properties, and streamlining of traditional methods (1). However, advanced application of MatEx is limited by the vast variability in part properties. In particular, the mechanical strength of MatEx-printed parts is yet to rival that of traditionally manufactured parts. Furthermore, a method to predict strength directly from the printing conditions is lacking.

MatEx involves extrusion of molten polymer material through a nozzle onto a build plate followed by rapid cooling and solidification. This deposition process is repeated layer by layer to construct a three-dimensional object consisting of a number of partially welded filaments. A number of three-dimensional computation fluid dynamics models have recently been developed to capture this deposition process (2-4). Gleadall et al. (5) employ a new computationally efficient method based on volume conservation; however, temperature and nonNewtonian effects are neglected from this approach. It has been shown that the feed velocity and temperature profile within the extruder are correlated $(6)$, and a method to predict temperature from the pressure drop is proposed. A review focusing on the thermal modeling is included in the chapter titled "Thermal Modeling of Material Extrusion Additive Manufacturing" in this book.

There are a large number of parameters that influence the part quality, such as build orientation, layer thickness, air gap, infill density, and feed rate (7). Moreover, the interfaces between adjacent filaments are likely to possess different material properties than the bulk of the filaments (8). Ultimately, the strength of a printed part relies on successful interdiffusion of the polymers across these filament-filament interfaces (9). However, the rapid cooling required for maintaining the structural integrity of the part means that interdiffusion is arrested at the onset of the glass transition. If the temperature of the weld reaches the glass transition temperature before a fully entangled network has formed across the interface, then the mechanical strength of the weld will be compromised (10).

Carefully calibrated infrared imaging has been developed to measure the temperature profile of the weld (11), and finite-element simulation methods have been employed to overcome limitations in spatial and temporal resolution (12). Although increasing the print temperature leads to longer welding times and can therefore increase the mechanical strength of the weld, bulk strength is not usually achieved (13), and the underlying reason remains unclear. Laser-assisted heating has been proposed as a method for improving welding (14); however, this technique cannot be used optimally without understanding the effects of temperature on the material microstructure.

Polylactic acid (PLA) and acrylonitrile butadiene styrene (ABS) are the two most-commonly used thermoplastics in MatEx. PLA has the advantage of 
biodegradability, so it is ideal for biomedical applications and, because of its lower printing temperature, is better suited to parts requiring fine details. Unlike ABS, PLA has a semicrystalline microstructure. Thus, during cooling, the polymers will nucleate and spherulites will grow depending on the conditions. It is suggested that interdiffusion must precede crystallization to ensure sufficient adhesion at the weld lines (15).

Crystallinity also plays a major role in the ultimate strength of thermoplastic parts. For example, there is a precedent for using postprinting thermal annealing to increase the crystal fraction, and consequently the strength, of printed parts (16). Without postprocessing, the degree of crystallinity can vary with print temperature, plate temperature, layer thickness, and even the color of the feedstock material (17-19). Furthermore, MatEx deformation rates are expected to be sufficiently large that the polymer molecules will become stretched and oriented during the deposition process (20). If the polymer backbone remains stretched at the onset of nucleation, then crystallization will be enhanced, leading to a significantly shorter crystallization time, as well as different crystal morphologies (21-22). Although this flow-induced crystallization (FIC) phenomenon has been extensively investigated for traditional polymer processing methods, there is much less research focusing on molecular deformation and FIC during MatEx. However, knowledge of how the crystal morphology, that is, both the degree of crystallinity and spherulite size, varies throughout a part and even throughout a single filament is crucial to understanding and ultimately controlling mechanical properties.

Recent in-situ Raman spectroscopy experiments have shown that FIC can occur under certain MatEx conditions for polycaprolactone (PCL) feedstock (23). A recently developed model is able to quantitatively predict the measured crystallization times (24) and highlights important features not captured by a single transient measurement of crystallization. In particular, the model shows that FIC leads to a dramatic cross-sectional variation of morphology in a single deposited filament, with smaller spherulites forming in an outer skin layer. Since FIC is expected to increase the availability of spherulites to form across the weld interface, therefore eliminating chain pull out as a failure mechanism, FIC may play a role in optimizing weld strength. Thus, the integration of experiments with accurate molecularly aware models of the crystallization process is essential to predicting part properties directly from printing conditions.

Unlike PCL, which reaches full space filling (maximum crystallinity) under typical MatEx conditions because of its low glass transition temperature (below room temperature), the crystallization kinetics of PLA may be arrested by its comparatively higher glass transition. In this chapter, we apply the previously developed model of McIlroy et al. (24) for PLA material properties to investigate the interaction between FIC and the glass transition during MatEx printing. The model contains only a single fitting parameter - all other parameters are derived directly from experimental measurements of the material properties - and predicts 
the nucleation density profile resulting from a combination of MatEx temperature history and flow effects. We then model a typical postprinting thermal annealing process to investigate spherulite growth from a fixed nucleation density. For the first time, a modeling approach for predicting the crystal morphology within an annealed MatEx-printed filament is compared to optical microscopy images for a range of printing conditions. The model reveals how feed rate and print temperature influence FIC during MatEx printing and appear as "templated" crystallinity after annealing, in agreement with experimental observations.

This chapter is organized as follows. First, we describe the experimental methods employed, including the techniques used to characterize PLA as well as the printing and imaging methodology. Second, we summarize the FIC model presented in ref (24). The Results section comprises details of the model parameters obtained from material characterization; the observed and predicted crystal morphology postprinting and postannealing is then presented. Finally, we discuss the model predictions and future directions.

\section{Experimental Methods}

Certain commercial equipment, instruments, or materials are identified in this chapter in order to specify the experimental procedure adequately. Such identification is not intended to imply recommendation or endorsement by the National Institute of Standards and Technology, nor is it intended to imply that the materials or equipment identified are necessarily the best available for the purpose.

\section{Material Characterization Sample}

The material used is the "natural" color Premium PLA Filament (Airwolf 3D) (25). The mass average molar mass of the sample is $103.3 \pm 0.4 \mathrm{~kg} / \mathrm{mol}$ with a dispersity of $1.64 \pm 0.05$ as measured by gel permeation chromatography of the sample dissolved in tetrahydrofuran, measured against polystyrene standards. Prior to material characterization and MatEx printing measurements, the filament is dried at $60^{\circ} \mathrm{C}$ under vacuum for at least $12 \mathrm{~h}$. Reported variation is 1 standard variation from 3 GPC injections.

\section{Linear Rheology}

Small-amplitude oscillatory rheology measurements are performed on a rheo-Raman microscope (26) using an $8 \mathrm{~mm}$ parallel plate geometry in a nitrogen 
atmosphere. Filament segments are loaded into the rheometer and pressed at $200^{\circ} \mathrm{C}$ to a gap of $1 \mathrm{~mm}$ prior to measurement. Samples are visually inspected using the optical microscope attachment to confirm that gas bubbles are not trapped in the polymer melt within the imaging window. Measurements are performed in the temperature range of $140-216^{\circ} \mathrm{C}$ using a strain of 0.05 .

\section{Optical Microscopy}

Optical imaging of isothermal crystallization processes is performed on an optical shear cell (CSS450, Linkam). Filament sections are melted at $200^{\circ} \mathrm{C}$ and pressed to a nominal thickness of $10 \mu \mathrm{m}$. Samples are then held for $180 \mathrm{~s}$ prior to cooling at a rate of $30^{\circ} \mathrm{C} / \mathrm{min}$ to the desired crystallization temperature $\left(30^{\circ} \mathrm{C} / \mathrm{min}\right.$ was chosen as the maximum cooling rate). Polarized optical imaging in transmission mode is performed on an Olympus BX-51 microscope using extralong working distance objectives.

\section{Raman Spectroscopy}

Raman spectroscopy measurements of isothermal crystallization processes are performed on the rheo-Raman microscope. The filament is loaded at $200^{\circ} \mathrm{C}$, pressed to a thickness of $0.8 \mathrm{~mm}$ in parallel plate geometry, and then held for $180 \mathrm{~s}$ to melt the polymer prior to cooling at a rate of $10^{\circ} \mathrm{C} / \mathrm{min}$ to the desired crystallization temperature. Raman spectra are measured using a $780 \mathrm{~nm}$ excitation wavelength with a power of $24 \mathrm{~mW}$ focused onto the sample using a 10x objective. Three exposures of $10 \mathrm{~s}$ each are averaged together for each recorded spectrum. The $\mathrm{C}=\mathrm{O}$ stretch region of the spectrum $1670-1850 \mathrm{~cm}^{-1}$ is analyzed using multivariate curve resolution using a constrained alternating least squares algorithm (MCR-ALS), which has been used previously to analyze polymer crystallization (27). The MCR-ALS algorithm is implemented in MATLAB (28) under the assumptions that there are two principal components with nonnegative intensities in the component spectra and nonnegative concentrations. Under these constraints, the relative concentration of one of the components varies from 0 at early times to 1 at the end of the crystallization process, which is identified as the relative crystallinity. 


\section{MatEx Printing and Imaging}

\section{Printing Conditions}

Samples were printed on an Axiom Direct Drive Dual (AirWolf3D) using a range of temperatures $\left(200,225\right.$, and $\left.250{ }^{\circ} \mathrm{C}\right)$ and print speeds $(10,30$, and $100 \mathrm{~mm} / \mathrm{s}$ ) amenable to the PLA used in the study. The selected print speeds correspond to filament feed rates of approximately $0.23,0.70$, and $2.3 \mathrm{~mm} / \mathrm{s}$ for a $2.85 \mathrm{~mm}$ diameter filament and 0.3-by-0.5-mm printed filament cross section. The sample geometry is similar to previously published work on infrared thermography $(11,13)$. For this work, measurements were made on samples with the following dimensions: $100 \mathrm{~mm}$ long ( $y$-axis), $4.8 \mathrm{~mm}$ tall ( $z$-axis, 16 layers at $0.3 \mathrm{~mm}$ ), and $0.5 \mathrm{~mm}$ wide ( $x$-axis, extruder diameter). The sample was printed $30 \mathrm{~mm}$ from the left of the build plate. To prevent the sample from detaching from the build plate during the printing process, $15 \mathrm{~mm}$ ( $y$-axis) by $15 \mathrm{~mm}$ ( $x$-axis) feet were added to the start and end of the first layer. The first layer is always printed at $30 \mathrm{~mm} / \mathrm{s}$ with $125 \%$ over extrusion. Subsequent layers are printed at the described speed and always printed in the same direction (front to back).

\section{Infra-red Imaging}

Temperature profiles were acquired using the procedure found here $(11,13)$, with minor modifications. Unlike previous work, regions-of-interest (ROIs) were selected algorithmically using the MATLAB ${ }^{\mathrm{TM}}$ edge detection function with a Canay filter. Default settings were sufficiently accurate to pick out the top edge of the print and ROIs were selected 5 pixels below the top edge for the center of the print layer, 15 pixels for the first sublayer, and 25 pixels for the second sublayer. The lens configuration and subsequent processing steps are detailed in the above work. ROIs were taken from the center of the print, $50 \mathrm{~mm}$ from the edge. The Axiom printer was modified by cutting a 3 in $(7.62 \mathrm{~cm})$ hole in the left panel to allow access for the IR lens and to prevent blocking of IR photons.

\section{Microtoming and Annealing}

Prior to microtoming, printed samples were cut in half and each half was further cut to approximately $1 \mathrm{~cm}$ in length using a wet saw. The cut sections were dried under a dynamic vacuum at $60{ }^{\circ} \mathrm{C}$. A $1-\mathrm{cm}$ section from each printing condition was annealed under a dynamic vacuum for $1 \mathrm{~h}$ at $140{ }^{\circ} \mathrm{C}$ to grow the spherulite size; the other $1-\mathrm{cm}$ section was used as received after printing. The 1 -cm sections were clamped directly and microtomed into $5 \mu \mathrm{m}$ to $8 \mu \mathrm{m}$ sections 
using a Histo Cryo $45^{\circ}$ diamond knife (DiATOME) on an EM UC7 (Leica); water was used to capture the sections. Microtomed sections were taken from the center of the print, $50 \mathrm{~mm}$ from the edge to match the IR ROIs. Cross sections were stored on microscope slides at $60{ }^{\circ} \mathrm{C}$ under dynamic vacuum until imaging.

\section{FIC Model for MatEx}

\section{MatEx Process Model}

To establish a fundamental representation of the key processes occurring during MatEx, the method of depositing a single filament onto the build plate is deconstructed into two distinct regions, as shown in Figure 1.

- $\quad$ Region I: The Flow Region

Here the melt flows through the hot nozzle and is deposited onto the build plate. Since the flow direction changes from the vertical to the horizontal (parallel with the build plate), the melt must deform to make a $90^{\circ}$ turn (Figure 2). The shape of the deposition is prescribed as elliptical to eliminate the need for a computationally expensive non-Newtonian Navier-Stokes calculation, and the velocity profile is given by conservation of mass. The polymers are deformed because of velocity gradients in the flow, and the deposition flow is assumed to be sufficiently fast that no polymer relaxation occurs. Furthermore, as assumed in previous work and considered a reasonable approximation (20), the temperature of the material in the nozzle and during the deposition process is assumed to be constant and above the melting point so that the material is fully amorphous.

- $\quad$ Region II: The Cooling and Crystallization Region

In this region, the melt cools and the polymer deformation relaxes in the absence of velocity gradients (i.e., we assume no further spreading occurs after deposition). Cooling of a single filament is assumed to be axisymmetric, and the boundary condition is prescribed by experimental observations. In this way nonisothermal effects are captured without the need for a computationally expensive thermal calculation. Once the temperature falls below the melting temperature $T_{M}$, the polymer spontaneously nucleates and spherulites begin to grow according to the Schneider rate equations. Crystallization is enhanced if there is residual stretch at the onset of nucleation. The computation is stopped near the glass transition.

This framework has been used in previous work for both amorphous polymers (10) and semicrystalline polymers (24). In this way, the flow and crystallization 
kinetics are decoupled. In this work, we do not consider the effect that deposition of multiple layers has on the crystallization kinetics.

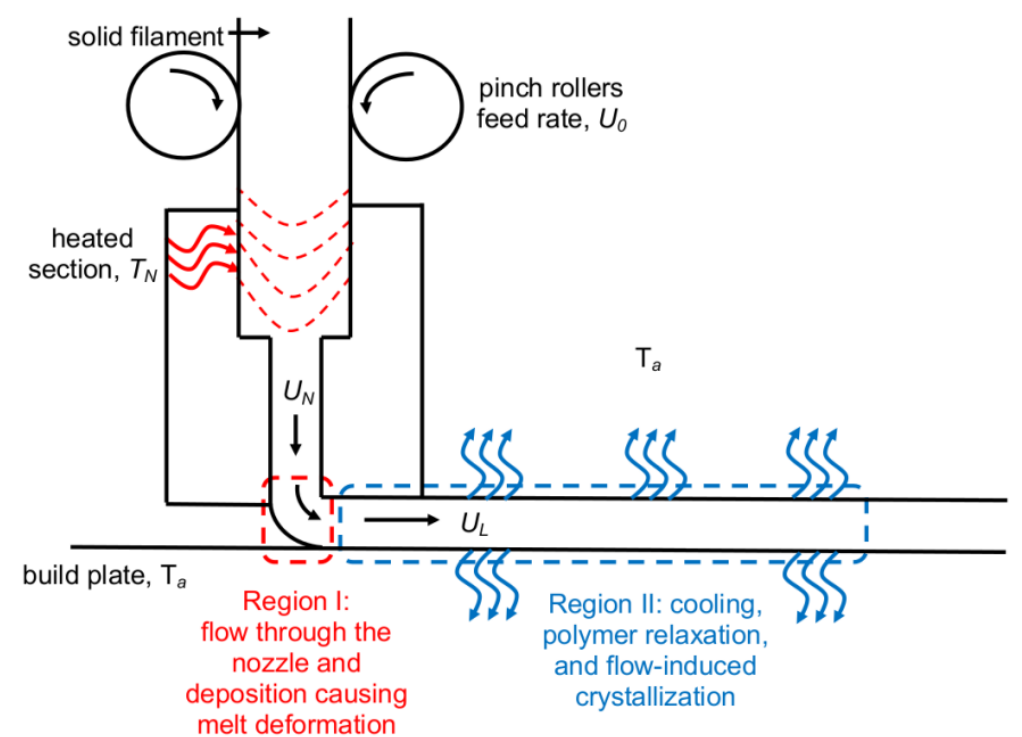

Figure 1. Schematic illustrating how flow and crystallization are decoupled in the MatEx model. Adapted with permission from ref (24). Copyright 2018 Wiley.
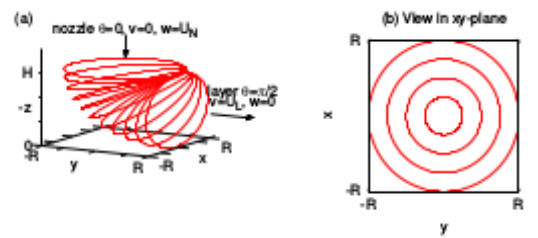
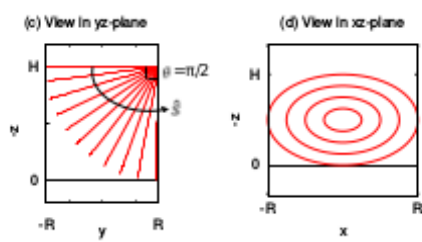

Figure 2. Shape of the deposited filament from four perspectives. The melt exits a circular nozzle, radius $R_{N}$, at speed $U_{N}$ and traces a smooth elliptical arc. The melt is deposited onto a build plate transformed into an elliptical cross section of height $H$. In a frame fixed with the nozzle the build plate moves at speed $U_{L}$. The shape of the deposit is parameterized by angle $\theta$. Adapted with permission from ref (20). Copyright 2017 AIP.

For completeness, in the following sections, we briefly describe the key equations that form the basis of this MatEx process model. Further details of the flow model are given in ref (20), and the cooling and crystallization model is discussed in detail in ref (24). 


\section{Constitutive Model}

To describe the deformation and relaxation of the polymer microstructure, we employ the Rolie-Poly model (29), which provides a single one-mode constitutive equation for the stress tensor based on the Doi-Edwards tube model for linear monodisperse polymer melts (30).

A melt is characterized by its dimensionless entanglement number

$$
Z=\frac{M_{w}}{M_{e}}
$$

where $M_{w}$ is the molecular weight of the polymer chain, and $M_{e}$ is the molecular weight between entanglements. The relaxation time of a single entanglement segment at some reference temperature $T_{0}$ is denoted $\tau_{e}^{0}$.

The Rouse time and the reptation time of a polymer chain is then given by

$$
\begin{gathered}
\tau_{R}=\tau_{e}^{0} Z^{2} a(T), \\
\tau_{d}=3 \tau_{e}^{0} Z^{3}\left(1-\frac{3.38}{\sqrt{Z}}+\frac{4.18}{Z}-\frac{1.55}{\sqrt{Z}^{3}}\right) a(T),
\end{gathered}
$$

respectively, where $a(T)$ is the Williams-Landel-Ferry (WLF) equation (see ref (31)):

$$
\boldsymbol{a}(\boldsymbol{T})=\exp \left(-\frac{\boldsymbol{C}_{1}\left(\boldsymbol{T}-\boldsymbol{T}_{0}\right)}{T+C_{2}-T_{0}}\right)
$$

for constants $C_{1}$ and $C_{2}$.

The total stress in a polymer melt is given by

$$
\sigma=-p I+G_{e}(A-I)+2 \mu_{s}\left(K+K^{T}\right)
$$

where $p$ is the isotropic pressure, $G_{e}$ is the plateau modulus, and $\mu_{s}$ is the contribution to the background viscosity from Rouse modes shorter than $M_{e}$, which is usually defined as the following (see ref (32)):

$$
\mu_{s}=\frac{\pi^{2}}{12} \frac{G_{e} \tau_{R}}{Z} \text {. }
$$

The identity tensor is denoted $\boldsymbol{I}$, and the velocity gradient tensor is given by $K_{i j}=$ $\partial u_{i} / \partial x_{j}$ for some velocity field $u_{i}$. Finally, the polymer configuration tensor is defined by

$$
A=\frac{<R R>}{R_{g}^{2}}
$$

where $\boldsymbol{R}$ is the end-to-end vector of a single polymer chain and $R_{g}$ is the polymer's root mean squared radius of gyration.

Tensor $\boldsymbol{A}$ obeys the so-called Rolie-Poly equation:

$$
\frac{D A}{D t}=\left(K \cdot A+A \cdot K^{T}\right)-\frac{1}{\tau_{d}(T)}(A-I)-\frac{2}{\tau_{R}(T)}\left(1-\frac{1}{\Lambda}\right)\left(A-\frac{\beta}{\Lambda}(A-I)\right),
$$


where the stretch of a polymer chain is denoted

$$
\Lambda=\sqrt{\frac{\operatorname{tr} A}{3}}
$$

for the trace of tensor $\boldsymbol{A}$. The convective constraint release parameter $\beta$ is set to 0.5 to ensure a monotonic constitutive curve. The material derivative is given by $\frac{D}{D t}=\frac{\partial}{\partial t}+(\boldsymbol{u} \cdot \nabla)$. The first term of the Rolie-Poly equation represents deformation due to velocity gradients, whereas the last two terms represent the two relaxation mechanisms: reptation along the polymer tube and Rouse relaxation of the polymer chain, respectively.

\section{Region I: Molten Flow}

\section{Flow through the Nozzle}

Assuming that the nozzle is axisymmetric, in cylindrical polar coordinates, the velocity profile is given by

$$
\boldsymbol{u}=(\mathbf{0}, \mathbf{0}, \boldsymbol{w}(\boldsymbol{r}))
$$

for radial coordinate $r$. Assuming steady-state flow, conservation of momentum becomes

$$
\frac{d p}{d z}=\frac{1}{r} \frac{\partial}{\partial r}\left(r \sigma_{r z}\right)
$$

where the shear stress is given by

$$
\sigma_{r z}=G_{e} A_{r z}+\mu_{s} \frac{\partial w}{\partial r}
$$

The polymer orientation $A_{r z}$ is calculated by the Rolie-Poly equation (Eq 7), and the pressure gradient $d p / d z$ is chosen to give a prescribed radially averaged extrusion velocity

$$
U_{N}=\int \frac{w(r)}{\pi R_{N}^{2}} d^{2} r, \quad(11)
$$

where $R_{N}$ is the nozzle radius. We assume no slip at the nozzle walls such that $w=0$ at $r=R_{N}$. This calculation gives an initial condition for the deposition flow.

For example, Figure 3 shows the cross-sectional stretch profile during flow through the nozzle for print conditions $U_{N}=100 \mathrm{~mm} / \mathrm{s}$ and $T_{N}=200^{\circ} \mathrm{C}$. The nozzle radius is $R_{N}=0.25 \mathrm{~mm}$. The polymer molecules become more stretched near to the nozzle walls due an increase in the shear rate. 


\section{Deposition Flow}

The cross section of the deposited filament is given by an ellipse with prescribed thickness $H$ and width $W=2 R_{N}$. The shape of the corner region is assumed to trace a smooth arc from the nozzle exit to the build plate, as shown in Figure 2. In this way, the velocity profile and the polymer stress are decoupled during deposition, eliminating the need for a full non-Newtonian calculation of conservation of momentum.

In Cartesian coordinates, the velocity profile is then given by

$$
\boldsymbol{u}=(\mathbf{0}, \boldsymbol{v}, \boldsymbol{w}),
$$

and the changing flow direction is written as

$$
s=\sin \theta \hat{e}_{y}+\cos \theta \hat{e}_{z}
$$

where $\theta \in[0, \pi / 2]$ denotes the angle between the nozzle exit and the deposited filament cross section (Figure 2 ). In order to conserve mass, we require

$$
v(\theta)=U_{N} \frac{A(\theta)}{\pi R_{N}^{2}} \sin \theta ; w(\theta)=U_{N} \frac{A(\theta)}{\pi R_{N}^{2}} \cos \theta
$$

where $A$ is the area of the cross section at angle $\theta$.

For steady-state deposition flow, which is sufficiently faster than polymer relaxation, the Rolie-Poly equation reduces to

$$
(\boldsymbol{u} \cdot \nabla) A=K \cdot A+A \cdot K^{T} .
$$

This calculation gives an initial deformation profile for the cooling and crystallization that occurs in Region II.

For example, Figure 3 shows the cross-sectional stretch profile after deposition flow for print conditions $U_{N}=100 \mathrm{~mm} / \mathrm{s}$ and $T_{N}=200{ }^{\circ} \mathrm{C}$. The height of the deposited filament is $H=0.3 \mathrm{~mm}$, and the width is $W=2 R_{N}=0.5 \mathrm{~mm}$. The polymer becomes more stretched in the bottom half of the cross section due to the $90^{\circ}$ turn combined with the assumption that the flow is faster than polymer relaxation.

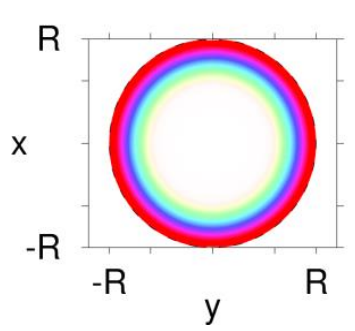

\section{6}

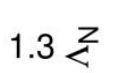

1

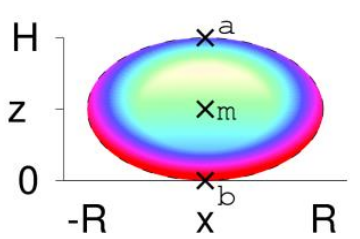

Figure 3. Polymer stretch profile $\Lambda$ in the nozzle cross section (left) and the deposited filament cross section (right). This stretch profile provides the initial condition for the crystallization calculation. 


\section{Region II: Cooling, Relaxation, and Crystallization}

\section{Cooling and Polymer Relaxation}

Once the filament has been deposited, the velocity gradients become zero (i.e., $\boldsymbol{K}=0$ ) and the polymer microstructure begins to relax via

$$
\frac{d A}{d t}=-\frac{1}{\tau_{d}(T)}(A-I)-\frac{2}{\tau_{R}(T)}\left(1-\frac{1}{\Lambda}\right)\left(A-\frac{\beta}{\Lambda}(A-I)\right) .
$$

Simultaneously, the filament begins to cool. Assuming axisymmetric cooling, the temperature profile in cylindrical polar coordinates $T \equiv T(t, r)$ is given by

$$
\frac{\partial T}{\partial t}=\alpha \frac{1}{r} \frac{\partial}{\partial r}\left(r \frac{\partial T}{\partial r}\right)+\frac{H_{f}}{C_{p}} \dot{\xi},
$$

where $\alpha$ is thermal diffusivity, $H_{f}$ is the latent hear of crystallization, $C_{p}$ is the specific heat capacity, and $\dot{\xi}$ is the crystallization rate. The thermal parameters $\alpha$, $H_{f}$, and $C_{p}$ are assumed to be constant for simplicity, as in ref (24).

The boundary condition at the free-surface $o$

$\mathrm{f}$ the deposited filament $T_{\text {surf }}(t)$ is prescribed by the infrared imaging measurements detailed in the Experimental Methods section and is discussed in more detail in Results I.

FIC

Once $T<T_{M}$, the polymer will nucleate and crystals will grow. Polymer nucleation and crystallization kinetics are well described by the Schneider rate equations (see ref (33)):

$$
\begin{array}{cl}
\dot{\phi}_{3}=8 \pi \dot{N}(T), & \left(\phi_{3}=8 \pi N\right), \\
\dot{\phi}_{2}=G(T) \phi_{3}, & \left(\phi_{2}=8 \pi R_{t o t}\right), \\
\dot{\phi}_{1}=G(T) \phi_{2}, & \left(\phi_{1}=S_{t o t}\right), \\
\dot{\phi}_{0}=G(T) \phi_{1}, \quad\left(\phi_{0}=V_{t o t}\right)
\end{array}
$$

where $N$ is the total number of nuclei per unit volume, $R_{\text {tot }}$ is the total radius, $S_{\text {tot }}$ is the total surface area, and $V_{\text {tot }}$ is the total volume of nuclei. To correct for impingement we use the Avrami equation (see ref (34))

$$
\phi_{0}=-\ln \left(1-\xi_{g}\right) \text {, }
$$

where $\xi_{g}$ is the degree of space filling. The degree of space filling is related to the degree of crystallinity via $\xi=V_{\max } \xi_{g}$, where $V_{\max }$ is the maximum degree of crystallinity. 
The crystal growth and nucleation rates are temperature dependent and denoted $G$ and $\dot{N}$, respectively. Under quiescent conditions, the nucleation rate is given by

$$
\dot{N}_{q}(T)=\dot{N}_{q}^{0}(T)\left(1-\frac{N}{N_{q, \max }}\right),
$$

where $\dot{N}_{q}^{0}(T)$ is the quiescent nucleation rate and the maximum number of nuclei that are allowed due to saturation effects is given by $N_{q, \max }$.

In the case of flow, the total number of nuclei is written as a linear combination of the quiescent nuclei plus flow-induced nuclei. Thus, the nucleation rate that enters the Schneider rate equations is given by

$$
\dot{N}=\dot{N}_{q}+\dot{N}_{f}
$$

where

$$
\dot{N}_{f}(T, \Lambda)=\dot{N}_{q}^{0}(T) \eta\left(\Lambda^{4}-1\right),
$$

as determined by kinetic Monte-Carlo simulations (22). Here, $\eta$ is the single fitting parameter that allows us to tune the number of extra nuclei due to flow effects. The Rolie-Poly model is thus coupled to the crystallization kinetics via the polymer stretch $\Lambda$.

\section{Results}

\section{Results I: Material Characterization and Model Parameters}

In this section we determine from material characterization of PLA the various properties required for the general FIC model described above. These material properties are summarized in Table 1 . The model is thus reduced to a single fitting parameter. A simple test based on the stretch relaxation time allows us to determine the conditions under which FIC will occur; these results are summarized in Table 2.

\section{Stretch Relaxation and Surface Cooling}

In order to determine if FIC is possible under the prescribed MatEx conditions, a simple test has been proposed (24), where the stretch relaxation time $\tau_{R}$ is compared to the time taken to reach to melting point $t_{M}$. We detail this procedure for PLA filament rheology here.

Due to the nonisothermal conditions of the MatEx process, the temperaturedependent rheology must be considered. In particular, the constitutive model 
requires the temperature dependence of the reptation time and Rouse time, as determined by the WLF equation, as follows. First a time-temperature superposition of the linear rheology data $\left(G^{\prime}, G^{\prime \prime}\right)$ is performed to create a master curve at reference temperature $T_{0}$. The two constants $C_{1}$ and $C_{2}$ appearing in the WLF equation are then obtained by fitting the exponential function to the shift factors required for superposition.

Fitting the same linear rheology data to the Likhtman and McLeish model (35) using Reptate software (36) yields the entanglement molecular weight $M_{e}$, the plateau modulus $G_{e}$, and the relaxation time of one entanglement segment $\tau_{e}^{0}$ at the reference temperature $T_{0}$. Thus, the entanglement number for this PLA feedstock is found to be $Z=25$, and the Rouse time and the reptation time can be computed at any temperature above the glass transition temperature $T_{g}$ (where both relaxation times diverge) via $\mathrm{Eq} 2$. The material properties derived from the linear rheology are listed in Table 1. 
Table 1. Material Properties for PLA Feedstock

\begin{tabular}{|c|c|c|c|c|}
\hline PLA Property & Notation & Value & Units & Source $^{a}$ \\
\hline Molecular Mass & $M_{w}$ & 103.3 & $\begin{array}{l}\mathrm{kg} \\
\mathrm{mol}^{-1}\end{array}$ & GPC \\
\hline Density & $\rho$ & 1200 & $\mathrm{~kg} \mathrm{~m}^{-3}$ & MAN \\
\hline $\begin{array}{l}\text { Melting } \\
\text { Temperature }\end{array}$ & $T_{M}$ & 168 & ${ }^{\circ} \mathrm{C}$ & MAN \\
\hline $\begin{array}{l}\text { Glass Transition } \\
\text { Temperature }\end{array}$ & $T_{g}$ & 60 & ${ }^{\circ} \mathrm{C}$ & MAN \\
\hline $\begin{array}{l}\text { Entanglement } \\
\text { Molecular Mass }\end{array}$ & $M_{e}$ & $4.0 \pm 0.4$ & $\begin{array}{l}\mathrm{kg} \\
\mathrm{mol}^{-1}\end{array}$ & LR \\
\hline Plateau Modulus & $G_{e}$ & $(8.7 \pm 0.9) \times 10^{5}$ & $\mathrm{~Pa}$ & LR \\
\hline $\begin{array}{l}\text { Entanglement Time } \\
\text { (at } T_{0} \text { ) }\end{array}$ & $\tau_{e}^{0}$ & $(6.7 \pm 0.7) \times 10^{-6}$ & $\mathrm{~S}$ & LR \\
\hline $\begin{array}{l}\text { Reference } \\
\text { Temperature }\end{array}$ & $T_{0}$ & 180 & ${ }^{\circ} \mathrm{C}$ & LR \\
\hline WLF Parameter & $C_{1}$ & $7.4 \pm 0.6$ & ${ }^{\circ} \mathrm{C}^{-1}$ & LR \\
\hline WLF Parameter & $C_{2}$ & $175.2 \pm 10.4$ & ${ }^{\circ} \mathrm{C}$ & LR \\
\hline \multirow{3}{*}{$\begin{array}{l}\text { Crystal Growth Rate } \\
\text { Parameters }\end{array}$} & $G_{\max }$ & $(4.9 \pm 0.1) \times 10^{-8}$ & $\mathrm{~m} \mathrm{~s}^{-1}$ & $\mathrm{OM}$ \\
\hline & $T_{S}$ & $122.4 \pm 0.3$ & ${ }^{\circ} \mathrm{C}$ & $\mathrm{OM}$ \\
\hline & $b_{g}$ & $23.1 \pm 0.5$ & ${ }^{\circ} \mathrm{C}$ & $\mathrm{OM}$ \\
\hline \multirow{4}{*}{$\begin{array}{l}\text { Nucleation Rate } \\
\text { Parameters }\end{array}$} & $n_{\max }$ & $(2.1 \pm 1.1) \times 10^{16}$ & $\mathrm{~m}^{-3} \mathrm{~s}^{-1}$ & $\mathrm{OM}$ \\
\hline & $b_{n}$ & $313 \pm 184$ & ${ }^{\circ} \mathrm{C}^{-2}$ & $\mathrm{OM}$ \\
\hline & $N_{q, \max }^{0}$ & $(9.66 \pm 0.75) \times 10^{17}$ & $\mathrm{~m}^{-3}$ & $\mathrm{OM}$ \\
\hline & $b_{\text {sat }}$ & $0.11 \pm 0.03$ & ${ }^{\circ} \mathrm{C}^{-1}$ & $\mathrm{OM}$ \\
\hline $\begin{array}{l}\text { Specific Heat } \\
\text { Capacity }\end{array}$ & $C_{p}$ & 1800 & $\begin{array}{l}\mathrm{J} \mathrm{kg}^{-1} \\
\mathrm{~K}^{-1}\end{array}$ & $(50)$ \\
\hline Heat of Fusion & $H_{f}$ & 93600 & $\mathrm{~J} \mathrm{~kg}^{-1}$ & (49) \\
\hline Thermal Diffusivity & $\alpha$ & $5.8 \times 10^{-8}$ & $\mathrm{~m}^{2} \mathrm{~s}^{-2}$ & $(50)$ \\
\hline
\end{tabular}

Note: $\mathrm{GPC}=$ gel permeation chromatography, MAN = manufacturer specifications, $\mathrm{LR}=$ linear rheology, $\mathrm{PC}=$ printing condition, $\mathrm{OM}=$ optical microscopy. 

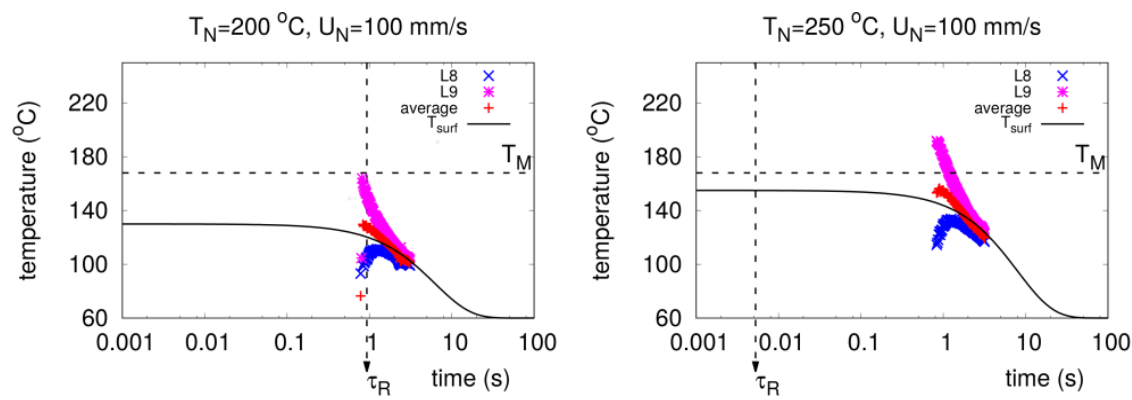

Figure 4. Cooling profile of filaments L9 and L8 measured using infrared imaging technique. The temperature profile at the weld is given by the average. The boundary condition imposed at the free surface of the filament is given by the line.

Figure 4 shows measurements taken using the infrared imaging technique detailed in the Experimental Methods section for print temperatures $T_{N}=$ $250{ }^{\circ} \mathrm{C}$ and $200{ }^{\circ} \mathrm{C}$ with print speed $U_{N}=100 \mathrm{~mm} / \mathrm{s}$. The temperature of the weld region is determined by the average of the two adjacent filaments (layers 8 and 9).

Under the assumption of axisymmetric cooling, the surface temperature of a single deposited filament is assumed to decay exponentially such that

$$
\boldsymbol{T}_{\text {surf }}(\boldsymbol{t})=\boldsymbol{T}_{\mathbf{0}} \exp (-\boldsymbol{A t})+\boldsymbol{T}_{\boldsymbol{a}},
$$

where $A$ is chosen to fit the experimental measurement of the weld line temperature. The cooling rate $1 / A$ is found to depend on both the print temperature and print speed. The ambient temperature $T_{a}$ is assumed to be equivalent to the build plate temperature.

According to one-dimensional modeling of a two-filament system (10), when a hot layer at $T_{N}$ is placed atop a cooled layer at $T_{a}$, the temperature of the weld region instantaneously jumps to the average temperature of the two filaments. Thus, we choose $T_{0}=\left(T_{N}-T_{a}\right) / 2$ in order to give $T_{\text {surf }}(t=0)=\left(T_{N}+T_{a}\right) / 2$. Eq 25 forms the boundary condition for the temperature model discussed in the previous section and is plotted in Figure 4. This modeling approach has proved successful in capturing MatEx-induced crystallization kinetics of a filament of PCL (19). The implications of this boundary condition are detailed in the Discussion section.

For this temperature model, we find that $T_{\text {surf }}(t=0)<T_{M}$ for all printing conditions. Thus, the time taken to reach the melting point is $t_{M}=0$, and nucleation will occur immediately. Since $t_{M}<\tau_{R}$ for all cases, any stretch induced by the MatEx printing flow will accelerate the nucleation rate at $t=$ 0 and lead to flow-enhanced crystallization for all the tested printing conditions. Table 2 summarizes the model parameters used for each printing condition. 
Table 2. Filament Cooling Rate Parameters, Time Above the Melt Temperature, and Rouse Times for All Print Conditions

\begin{tabular}{|c|l|l|l|l|l|l|}
\hline$T_{N}$ & $U_{N}$ & $A$ & $T_{\text {surf }}(0)$ & $t_{M}$ & $\tau_{R}\left(T_{\text {surf }}(0)\right)$ & $F I C$ \\
\hline 250 & 100 & 0.135 & 155.0 & 0 & 0.00058 & $\checkmark$ \\
\cline { 2 - 7 } & 30 & 0.139 & 155.0 & 0 & 0.00058 & $\checkmark$ \\
\cline { 2 - 7 } & 10 & 0.108 & 155.0 & 0 & 0.00058 & $\checkmark$ \\
\hline 225 & 100 & 0.192 & 142.5 & 0 & 0.00127 & $\checkmark$ \\
\cline { 2 - 7 } & 30 & 0.169 & 142.5 & 0 & 0.00127 & $\checkmark$ \\
\cline { 2 - 7 } & 10 & 0.113 & 142.5 & 0 & 0.00127 & $\checkmark$ \\
\hline \multirow{3}{*}{200} & 100 & 0.339 & 130.0 & 0 & 0.00324 & $\checkmark$ \\
\cline { 2 - 7 } & 30 & 0.234 & 130.0 & 0 & 0.00324 & $\checkmark$ \\
\cline { 2 - 7 } & 10 & 0.141 & 130.0 & 0 & 0.00324 & $\checkmark$ \\
\hline
\end{tabular}

\section{Quiescent Nucleation and Crystal Growth Rate}

In this section we determine the appropriate temperature-dependent forms of the quiescent nucleation rate $\dot{N}_{q}^{0}$ and the crystal growth rate $G$, which enter the Schneider rate equations (eq 19) through characterization of the crystallization kinetics.

Figure 5 shows the quiescent nucleation and crystal growth rate measured using the optical microscopy technique described in the Experimental Methods section. Crystal growth is fastest around $120^{\circ} \mathrm{C}$, and decays rapidly to zero near to the melting temperature and the glass transition temperature.

The temperature dependence of the crystal growth rate takes a Gaussian distribution of the form

$$
\boldsymbol{G}(\boldsymbol{T})=\boldsymbol{G}_{\text {max }} \exp \left(-\left(\frac{T-T_{s}}{\boldsymbol{b}_{g}}\right)^{2}\right),
$$

as shown in Figure 5. The constants $G_{\max }, T_{s}$ and $b_{g}$ are found by fitting Eq 25 to the optical microscopy measurements and are listed in Table 1 . We note that this equation is not the Hoffman-Lauritzen theory (37); it was chosen for agreement with experimental results.

The temperature dependence of the nucleation rate is determined by the existence of two competing effects, as described by Lauritzen and Hoffman (37). That is

$$
\dot{N}_{q}^{0}(T)=\frac{n_{\max }}{a(T)} \exp \left(\frac{b_{n}}{T-T_{M}}\right),
$$

as shown in Figure 5. Again, the constants $n_{\max }$ and $b_{n}$ are found by fitting Eq 26 to the optical microscopy measurements as well as data found in the literature (38) and are listed in Table 1. 

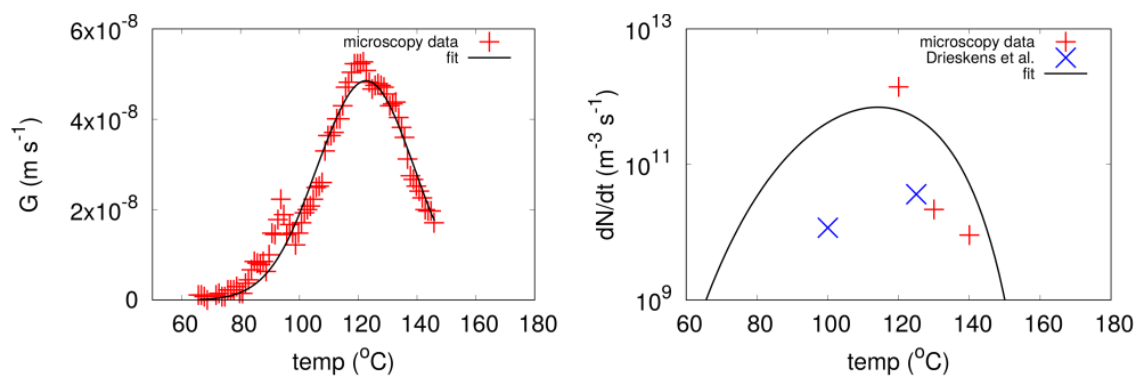

Figure 5. Crystal growth rate $G$ and quiescent nucleation rate $\dot{N}_{q}^{0}$ as measured using optical microscopy technique over a range of crystallization temperatures.

The fits used in the model are plotted as lines.

\section{Saturation Limit}

Figure 6 shows the evolution of the degree of space filling $\xi_{g}$, measured using the Raman spectroscopy technique described in the Experimental Methods section. The crystallization transients are measured under quiescent, isothermal conditions at three crystallization temperatures 90,100 , and $110{ }^{\circ} \mathrm{C}$. The maximum degree of crystallinity, as estimated using the univariate (peak intensity ratio) methods of Qin and Kean (39), is found to be between 0.2 and 0.25 in these cases. Thus, the degree of space filling is related to the crystal fraction via $\xi=V_{\max } \xi_{g}$, where $V_{\text {max }}=0.2$ to 0.25 . This data is used to establish the saturation limit $N_{q, \max }$ required in the FIC model.

If we relax the assumption of saturation and assume $N_{q, \max } \rightarrow \infty$, then the Schneider rate equations (Eq 19), along with the nucleation and crystal grow rates given by Eqs 25 and 26, are unable to capture the measured crystallization transients; in particular, crystallization is much slower at 100 and $110{ }^{\circ} \mathrm{C}$ than the kinetics predicted by the model, as shown in Figure 6 .
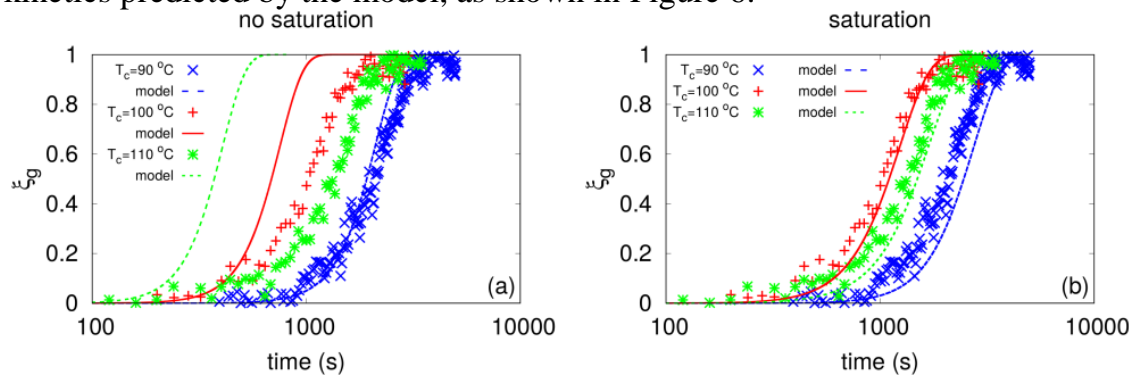
Figure 6. Degree of space filling measured over time via Raman spectroscopy technique for three crystallization temperatures (points). Model predictions neglecting saturation effects (left) and incorporating a finite saturation limit (right) are shown by the lines.

Defining a finite saturation limit $N_{q, \max }$ restricts the total number of nuclei that are created and therefore slows down crystal growth. We find that the saturation limit required to fit the experimental data is temperature dependent such that

$$
N_{q, \max }=\boldsymbol{N}_{q, \max }^{0} \exp \left(-\boldsymbol{b}_{s a t} T\right),
$$

where constants $N_{q, \text { max }}^{0}$ and $b_{\text {sat }}$ are given in Table 1 . The corresponding nucleation transient is shown in Figure 7.

The fact that the number of nuclei saturates well before the growth of crystallinity indicates that crystallization proceeds through heterogeneous nucleation, with more heterogeneous nucleation sites becoming active at lower temperatures. This behavior is well documented in the heterogeneous crystallization kinetics of polymers (40).

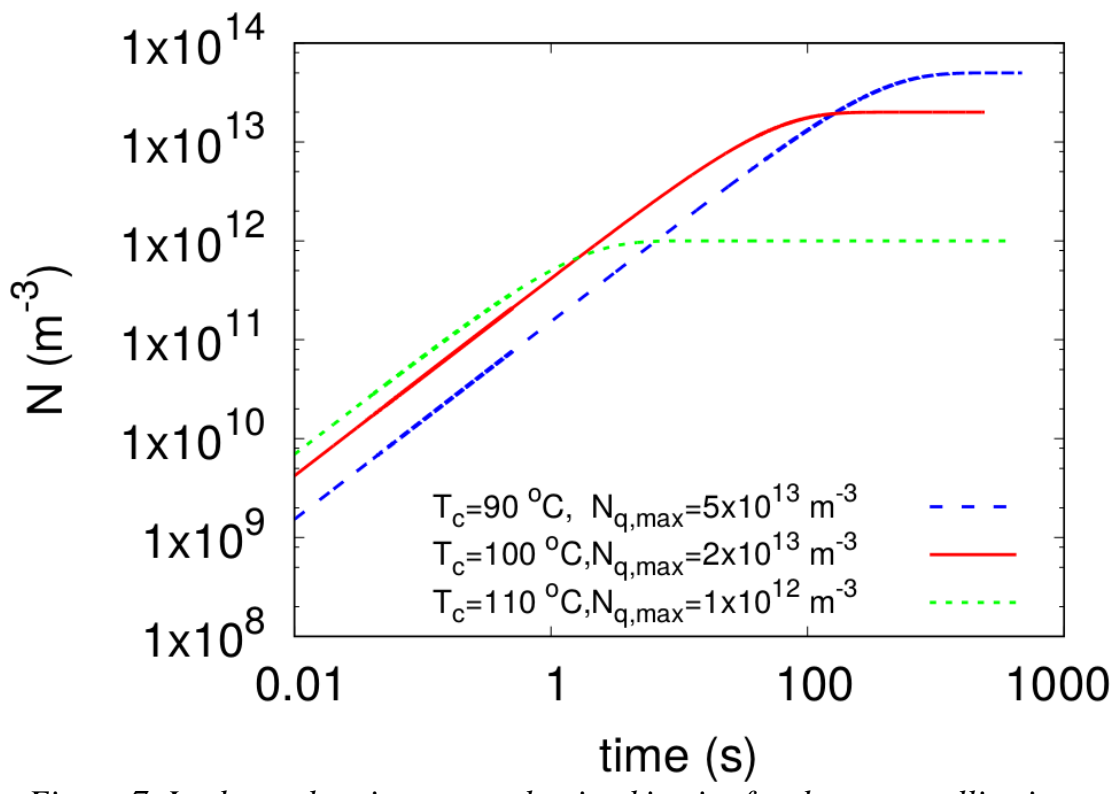

Figure 7. Isothermal, quiescent nucleation kinetics for three crystallization temperatures and corresponding temperature-dependent saturation limit

$$
N_{q, \max } \text {. }
$$




\section{Results II: Morphology of Printed Filaments}

In this section we compare the model predictions of the crystal morphology to optical microscopy images taken immediately after MatEx printing, where the polymer melt has nucleated but there is little crystal growth. The printed samples are then annealed and reimaged to investigate spherulite growth from the fixed number of nuclei induced by the printing conditions. Similar thermal annealing processes are used to increase the strength of printed parts by increasing the degree of crystallinity (18).

Although this annealing process leads to full space filling (i.e., maximum crystallinity), the size of the spherulites that grow depends on the nucleation density via the Schneider rate equations (eq 19). Since spherulite size is also expected to affect strength (43), full comprehension of the mechanical properties after annealing requires understanding how the nucleation density induced by the printing conditions varies cross-sectionally.

Thus, through validation of the cross-sectional nucleation density profile, the model can be employed to predict and quantify variations in spherulite size, which are difficult to measure experimentally. The model can then be used as a tool to explore variations in mechanical strength and failure mechanisms.

\section{Nucleation Density after Printing}

Figure 8 shows optical microscopy images of the printed filament cross sections for each of the nine printing conditions. The profiles of the individual filaments are concave towards the nozzle, which is expected when the nozzle extrusion height is less than the nozzle diameter (41). The profiles are also transparent under bright-field illumination. It is clear that little crystallization has occurred during the MatEx cooling period; any existing spherulites are smaller than the microscope resolution of approximately $1 \mu \mathrm{m}$. Since the model can predict how the nucleation density varies spatially in a printed cross section, we are able to quantify the degree of space filling achieved during printing. 


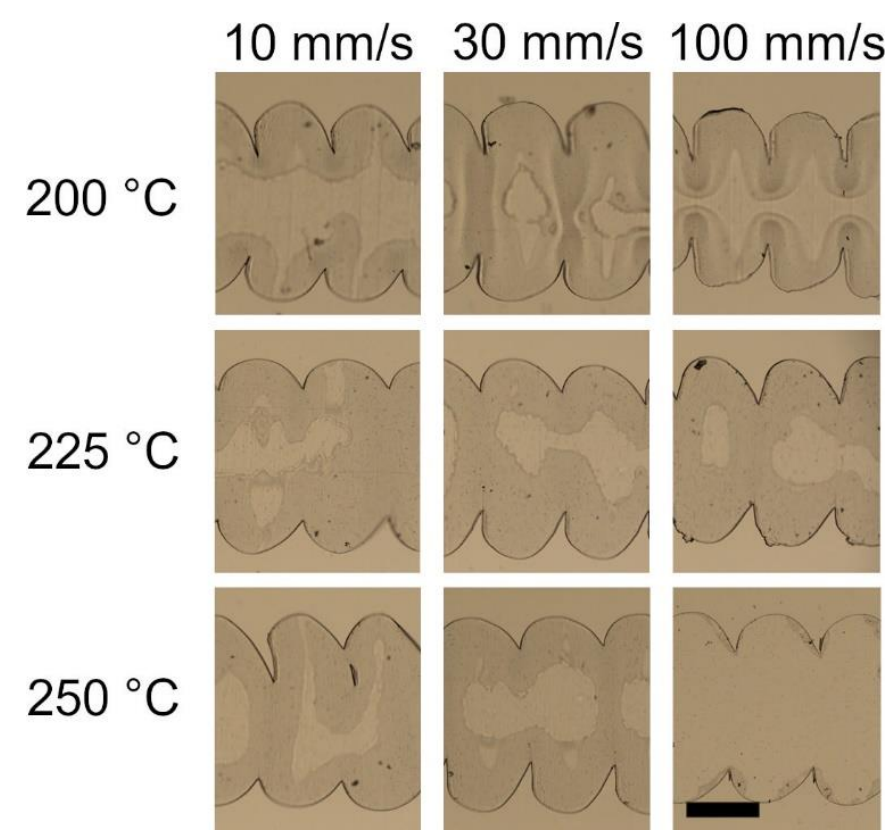

Figure 8. Images of the cross sections of the printed filament (pre-annealing) under bright-field illumination. The cross sections are dark in cross-polarized light. The scale bar is $200 \mu \mathrm{m}$.

Figure 9 shows the predicted nucleation density achieved after printing a single filament for each of the nine printing conditions. In each case, we observe a boundary layer near the free surface of the cross section having a larger nucleation density than the bulk of the filament; this boundary layer becomes more distinct for lower print temperatures and faster print speeds. In the following, we demonstrate how this high nucleation density boundary layer arises because of flow-enhanced crystallization occurring only at the surface of the deposited filament. 

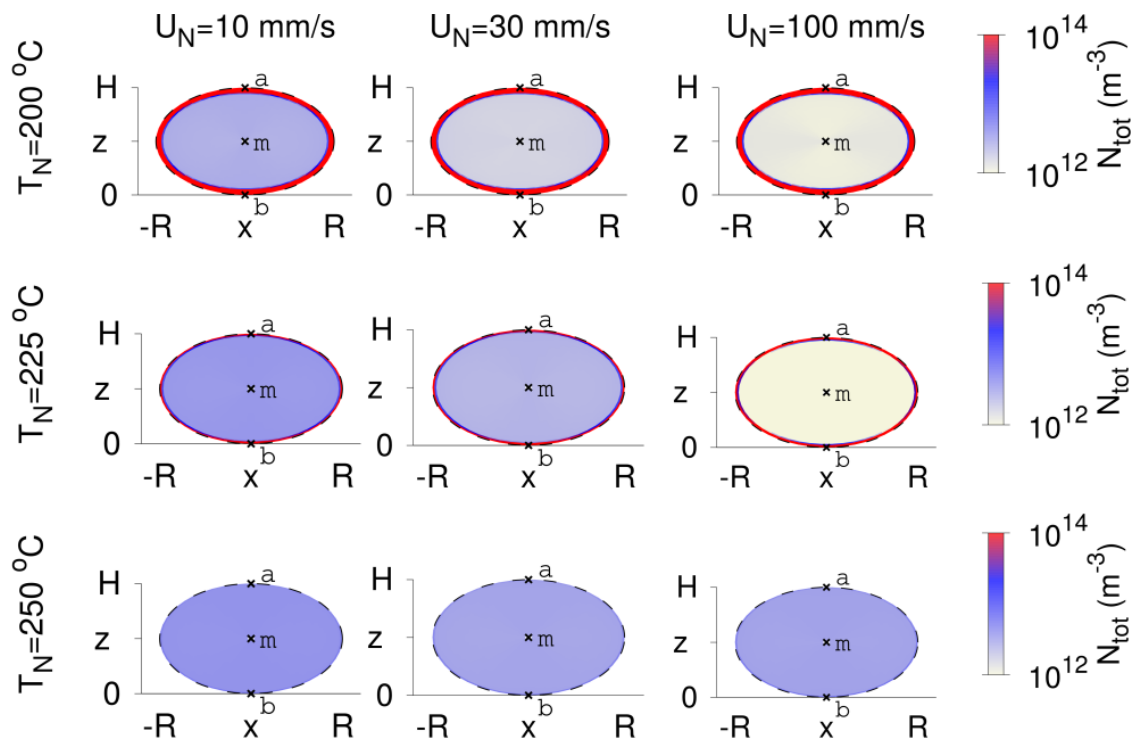

Figure 9. Predicted cross-sectional nucleation density for nine printing conditions. A boundary layer of flow-enhanced nuclei can be observed in each case, which becomes more distinct at lower print temperatures and faster print speeds.

To probe this spatially dependent behavior, we consider the kinetics at three locations on the filament cross section; the top (apex) $a$, the middle $m$ and the bottom $b$ (as illustrated in Figure 3). The surface locations $a$ and $b$ correlate to positions that reside in the weld regions after deposition of multiple filaments. In particular, Figure 10 shows how temperature, polymer stretch, the number of nuclei, and the degree of crystallinity evolve over time at these three locations until the temperature reaches the glass transition. These kinetics are presented for two printing conditions.

For both cases, the surface immediately drops below $T_{M}$ so that nucleation at the surface is enhanced by the polymer stretch determined by the MatEx flow. Model parameter $\eta$ is sufficiently large that the total number of nuclei surpasses the quiescent kinetics within the Rouse time, and nucleation stops once the polymer stretch has relaxed. On the other hand, the stretch imposed at the middle of the filament has sufficient time to relax before the melting temperature is reached. Thus, nucleation follows quiescent kinetics and is arrested once the temperature reaches the glass transition. Consequently, we find that the surface of the filament reaches a higher degree of space filling than the middle of the filament, which is unapparent in the optical microscopy images. 

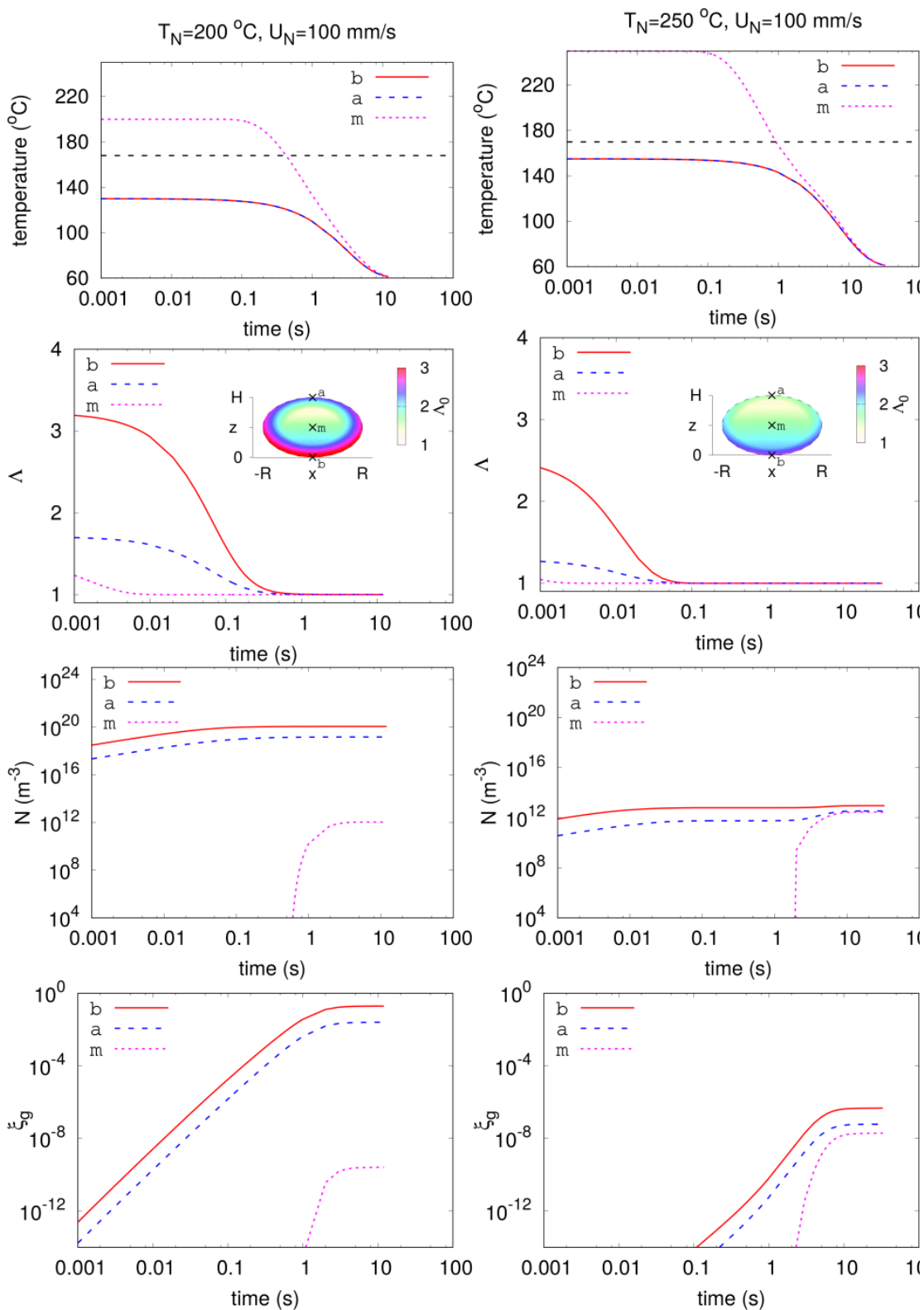

Figure 10. Evolution of the temperature, polymer stretch, number of nuclei and degree of space filling at the top $(a)$, middle $(m)$ and bottom $(b)$ of the cross section for two printing conditions. The initial stretch profile is shown as an inset. Flow-enhanced crystallization occurs only at the surface of the filament in both cases. 
Comparing $T_{N}=200$ and $250{ }^{\circ} \mathrm{C}$, we find that fewer nuclei are created at the surface at the higher print temperature, since there is less polymer stretch under this condition. Thus, the high nucleation density boundary layer shown in Figure 9 is less defined. More nuclei are created quiescently in the center of the filament at the higher print temperature due to a longer cooling rate $1 / A$. Figure 11 shows how the total number of nuclei created at the middle of the filament $m$ varies for each of the printing conditions. The total number of nuclei created increases with increasing cooling rates $1 / A$, as detailed in Table 2 for each case.

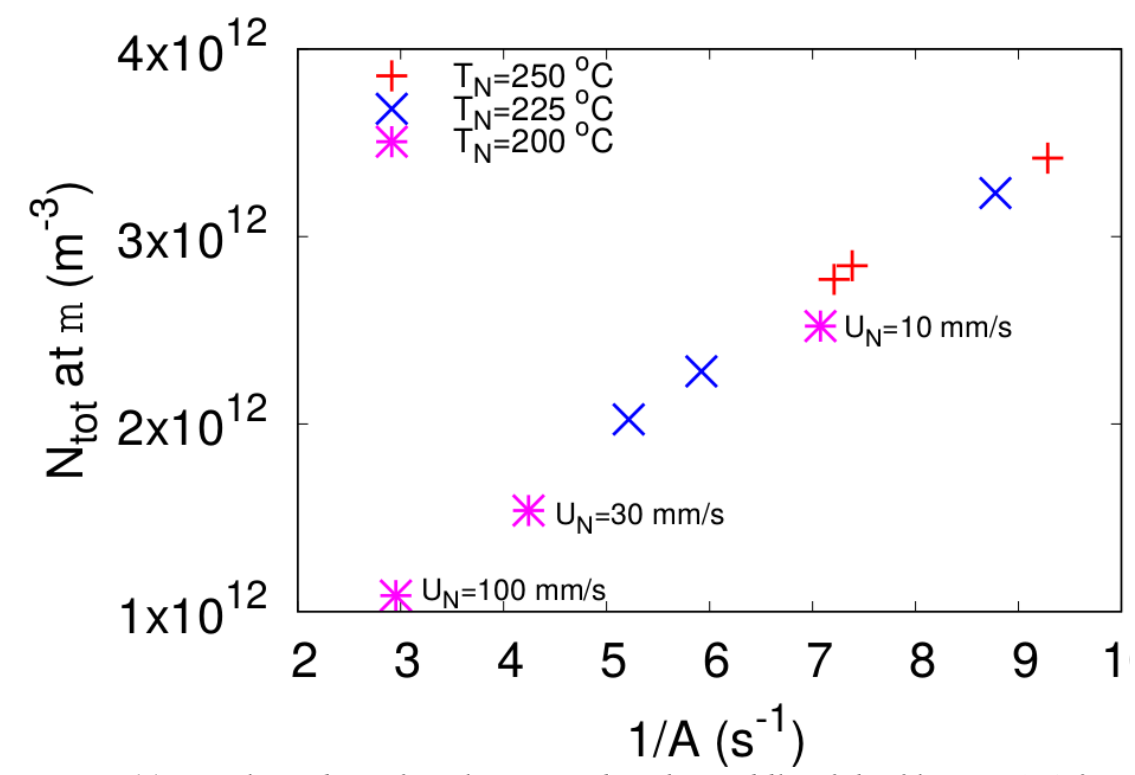

Figure 11. Total number of nuclei created at the middle of the filament $(m)$ for each of the nine printing conditions plotted against the cooling rate $1 / A$, as determined by a fit to infrared imaging measurements. The corresponding print speed for each case is detailed in Table 2.

Figure 12 shows how the choice of parameter $\eta$, which allows tuning of the number of extra nuclei created due to flow effects, affects the final degree of crystallinity for $T_{N}=200{ }^{\circ} \mathrm{C}$ and $U_{N}=100 \mathrm{~mm} / \mathrm{s}$. By increasing $\eta$, full space filling can be achieved at the surface. We choose $\eta=10^{8}$, as this yields a degree of space filling consistent with spherulites of the size of approximately $0.1 \mu \mathrm{m}$ in diameter, in other words, less than the resolution of the microscope (see Figure 12). 


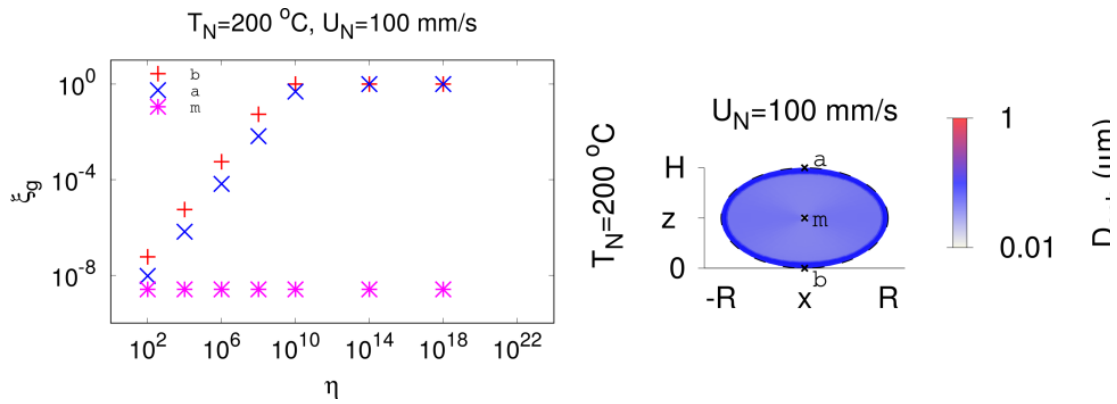

Figure 12. Degree of space filling achieved for different values of the model tuning parameter $\eta$. Since spherulites are too small for observation after printing (pre-annealing), we choose $\eta=10^{8}$. In this way, the (pre-annealed) spherulite sizes predicted by the model are smaller than the microscope resolution and space filling remains small $\xi_{g}<10^{-2}$.

\section{Spherulite Size Distribution after Annealing}

Figure 13 shows optical microscopy images of the microtomed cross sections after annealing at $140{ }^{\circ} \mathrm{C}$ for $1 \mathrm{~h}$. At this temperature, the nucleation rate is small so that the number of nuclei will remain approximately constant. The annealing process therefore increases the size of the semicrystalline domains templated into the printed part from the printing conditions. Under crossed polarizers, the microtomed sections are now strongly birefringent. As the temperature decreases and the print speed increases, the weld region appears to exhibit a lower birefringent intensity at the weld line.

We can characterize the weld region using image analysis to determine the width and optical intensity difference between the weld region and the filament midsection. A section of the image taken at a high magnification (Figure 14a) is first thresholded and binarized (Figure 14b), and then column averages of the pixel intensity $I$ in each image are plotted as a function of distance and fit to a Gaussian profile

$$
\boldsymbol{I}=\boldsymbol{I}_{0}-\boldsymbol{I}_{A} \exp \left(-\left(\frac{x-x_{0}}{w_{I}}\right)^{2}\right)
$$

where $I_{0}$ is the intensity of the region far away from the weld, $I_{\mathrm{A}}$ is the intensity loss at the center of the weld located at position $x_{0}$, and $w_{\mathrm{I}}$ is the peak width. An example of the Gaussian fit is shown in Figure 14c. For all printing conditions, the full-width at half maximum $\left(\mathrm{FWHM}=2 \sqrt{2 \ln 2} w_{I}\right)$ of the weld region is in the range of 35 to $52 \mu \mathrm{m}$, independent of temperature or feed rate. The relative intensity loss $I_{\mathrm{A}} / I_{0}$ is shown in Figure 15, which shows that the highest intensity loss occurs at the printing temperature of $200{ }^{\circ} \mathrm{C}$ and higher feed rates. This loss 
in intensity is not due to a lower crystallinity in the weld region; spot measurements of the Raman spectrum in the weld region and in the middle of the printed filament indicate negligible differences in the spectrum for a given microtomed section. This indicates that the crystallinity in the weld is not significantly different from the bulk. The relative intensity loss is therefore higher because of the presence of crystalline domains in the weld region that are below the diffraction limit (approximately $1 \mu \mathrm{m}$ ) for optical imaging.

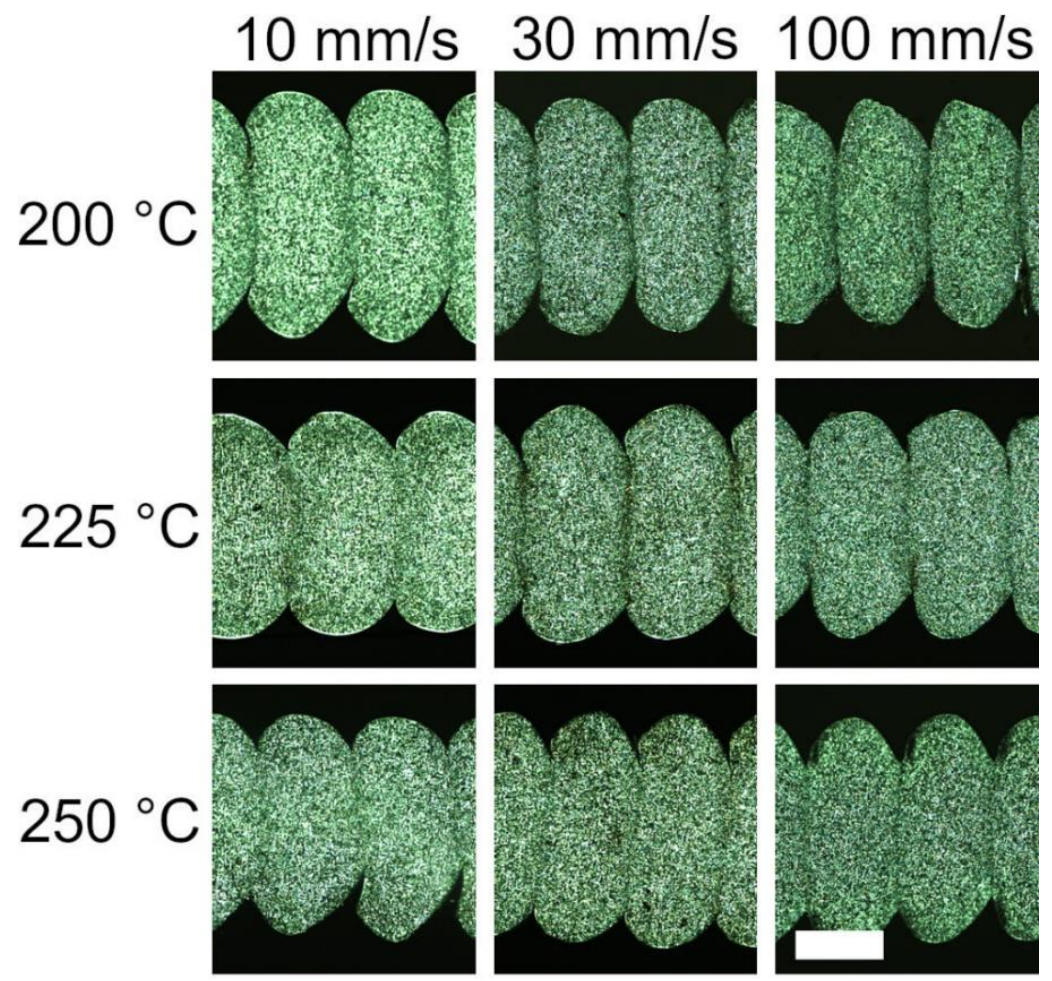

Figure 13. Images of the annealed cross sections of the prints using crosspolarized illumination. The cross sections are dark in cross-polarized light, with the polarizer and analyzer oriented at $45^{\circ}$ to the image. The scale bar is $200 \mu \mathrm{m}$. 
a)
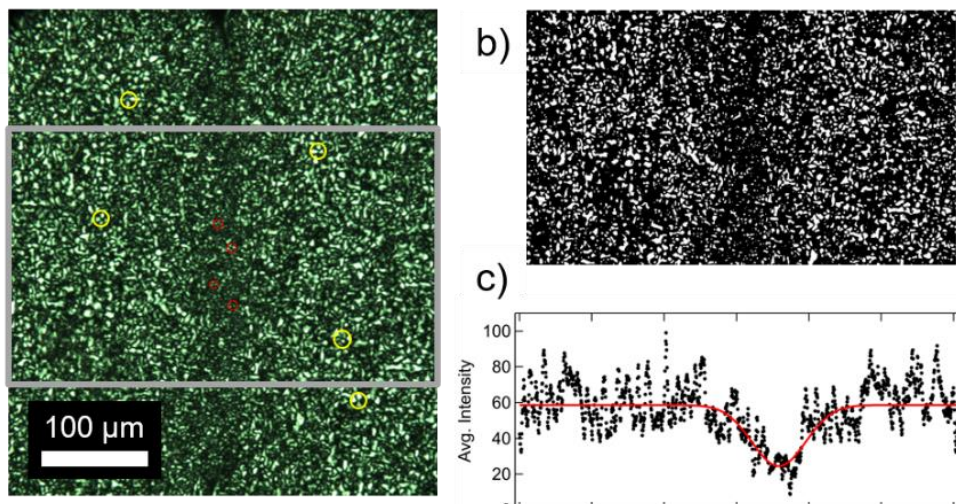

c)

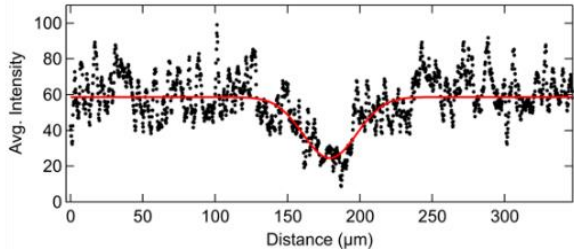

Figure 14. (a) Annealed cross section of the part printed at $200^{\circ} \mathrm{C}$ and $10 \mathrm{~mm} / \mathrm{s}$. Spherulites within $50 \mu \mathrm{m}$ of the weld are outlined with a red circle, and spherulites outside the weld region are indicated with a yellow circle.

The grey box indicates the region selected for intensity analysis. (b) The thresholded, binarized image of the selected region. (c) The pixel intensity as a function of distance. The points indicate the average pixel intensity and the line indicates the Gaussian fit to the intensity profile.

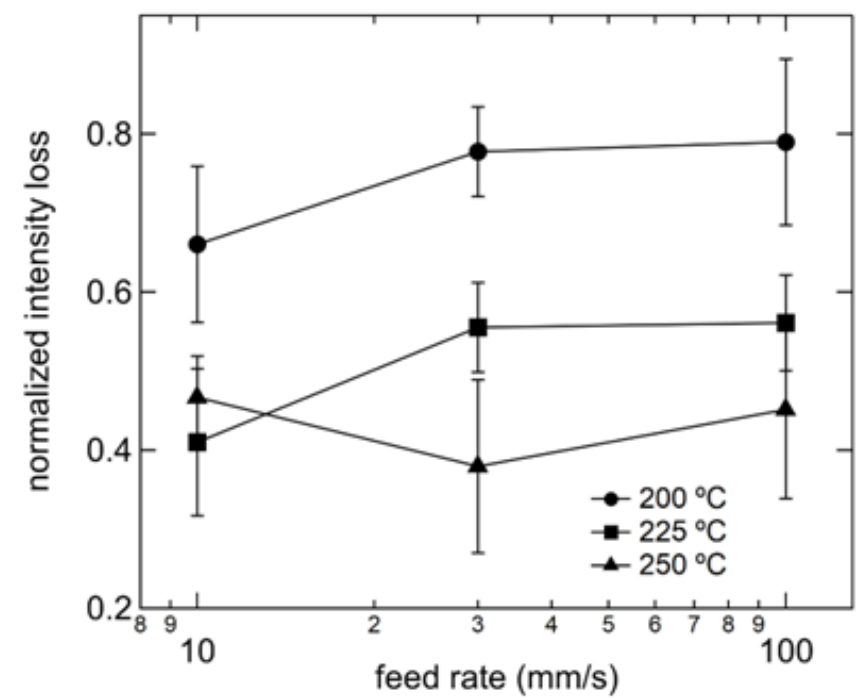

Figure 15. Normalized birefringent intensity loss at the weld versus feed rate. The error bars indicate the standard deviation based on measurements of four welds at each printing condition. 
Spherulites can be measured by manually fitting regions where a Maltese cross pattern is observed in the image, in both the weld region and towards the middle of the printed filament, examples of which are shown Figure 14a. Measurements of the spherulite diameter from polarized optical microscopy are shown in Figure 16. The spherulites in the middle of the printed filament decrease in size at higher feed rates and lower temperatures, and under all conditions, the spherulites near the weld are smaller than those in the middle of the printed filament. At the lowest printing temperature, the spherulites at 30 and $100 \mathrm{~mm} / \mathrm{s}$ in the weld region begin to look more granular, and the Maltese cross pattern is no longer clearly observed. The average diameter of the measurable spherulites in that region is approximately $5 \mu \mathrm{m}$, but we extend the lower error bar to zero to indicate that crystalline domains below the optical resolution limit are present.

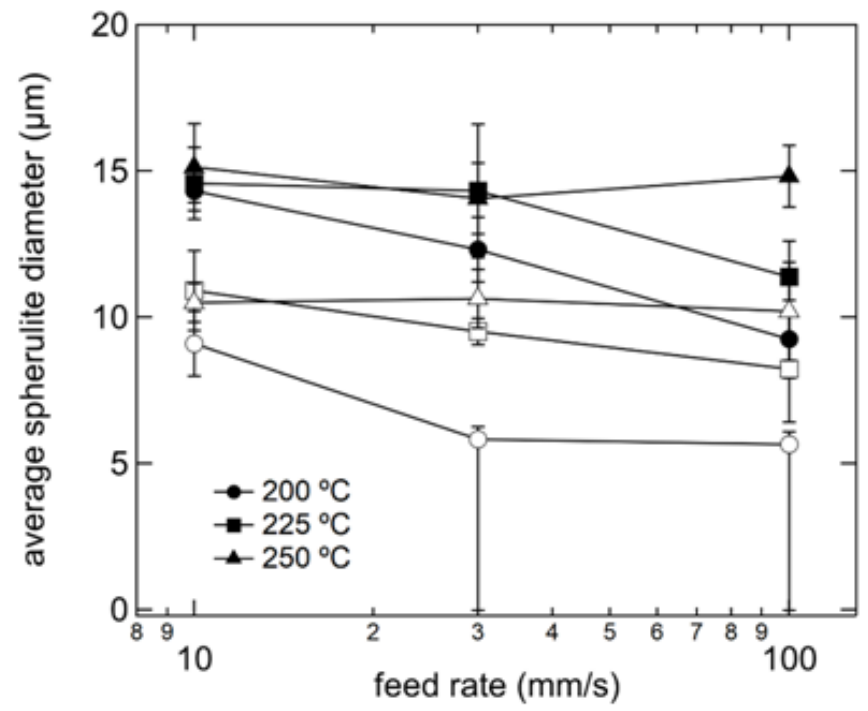

Figure 16. Average spherulite diameter measured from optical imaging versus feed rate. Filled symbols indicate spherulites measured towards the middle of the filament, and open symbols indicate spherulites within $50 \mu \mathrm{m}$ of the weld.

The error bars indicate the standard distribution from an average of five spherulites.

Using the crystal morphology induced by each printing condition as an initial condition, the model is used to simulate this annealing process; the temperature is instantaneously set to $T=140^{\circ} \mathrm{C}$, and the nucleation rate is set to $\dot{N}=0$ in the Schneider rate equations (Eq 19). Thus, spherulites will grow from the fixed nucleation density created during the MatEx cooling period; this nucleation density is cross-sectionally nonuniform (see Figure 9) due to a combination of 
temperature history and flow effects. Impingement of the spherulites is accounted for via the Avrami equation (Eq 20. The model predicts that uniform space filling is achieved across the filament during the annealing process, in agreement with the experimental observation.

Figure 17 shows the corresponding spherulite diameter $D_{s p h}$ after $1 \mathrm{~h}$ of annealing for each printing condition. We find qualitative agreement with the optical microscopy images, with much smaller spherulites found in the weld regions for all printing conditions. The model demonstrates that this crosssectional variation in morphology arises due to FIC at the surface of a deposited filament. Note that this boundary layer cannot be reproduced without enhanced nucleation due to stretch. That is, if $\eta$ is set to zero, then a similar number of nuclei are created at the surface and the middle of the filament despite the surface spending a significantly longer time below $T_{M}$; during this (early) time the temperature at the surface is high enough that the nucleation rate in this region is small. Thus, the final nucleation density at $T_{g}$ is not significantly different at $a, b$, or $m$ and does not lead to a difference in spherulite size after annealing, as shown in the experiments.
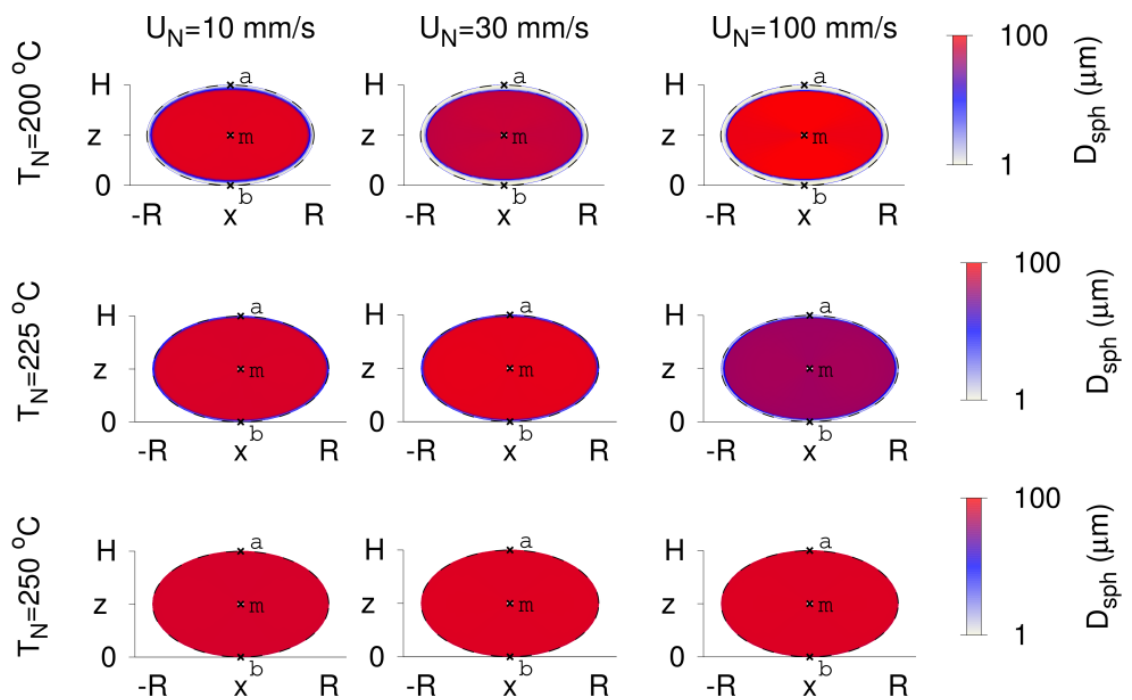

Figure 17. Predicted cross-sectional spherulite diameter after annealing for $1 \mathrm{~h}$ at $140{ }^{\circ} \mathrm{C}$ for each of the nine printing conditions. A flow-enhanced boundary layer of smaller spherulites can be observed in each case, which becomes more distinct at lower print temperatures and faster print speeds.

Quantitatively, Figure 18 shows how spherulite diameter $D_{s p h}$ varies with $z$, in other words, between the middle and weld lines of printed filaments (note that the results of a single filament are repeated to demonstrate a two-filament wall of 
height $600 \mu \mathrm{m})$. As seen in the experiments, the smallest spherulites $(\approx 5 \mu \mathrm{m})$ are produced at the lowest print temperature $T_{N}=200^{\circ} \mathrm{C}$ and the fastest print speeds $U_{N}=30 \mathrm{~mm} / \mathrm{s}$ and $100 \mathrm{~mm} / \mathrm{s}$. Spherulites smaller than the microscope resolution are also present in the center of the flow-enhanced boundary region. This boundary region is approximately $50 \mu \mathrm{m}$ in width and independent of print speed, in agreement with the experimental measurements. At higher print temperature $T_{N}=250{ }^{\circ} \mathrm{C}$, the spherulites in the weld region are much closer in size to the spherulites created in the bulk so that the flow-enhanced boundary layer is much less distinct.
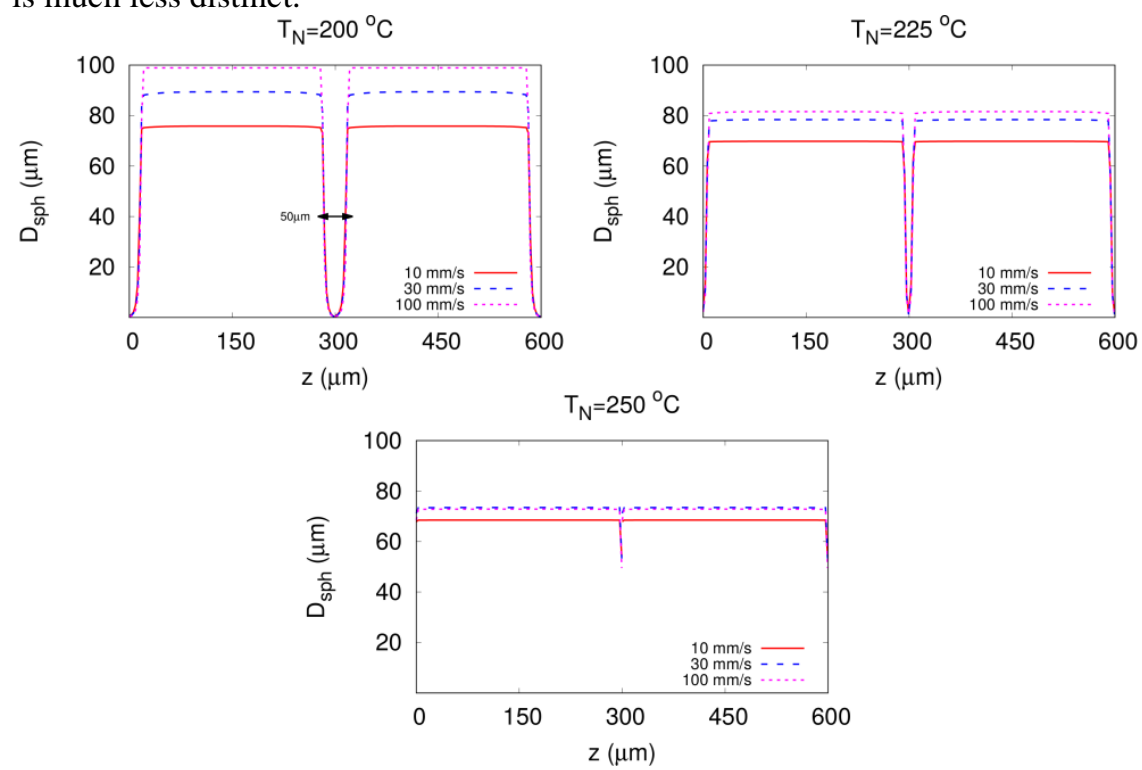

Figure 18. Predicted spherulite size as a function of $z$ for two filaments placed adjacently. The thickness of the boundary layer is independent of feed rate and increases with decreasing temperature.

The predicted diameter of spherulites residing in the bulk of the filament is quantitatively larger than the measured spherulite size. We believe this to be a result of further nucleation during the subsequent heating/cooling cycles that occur when multiple filaments are deposited to create the wall; for example, ref (42) shows that multiple layers rise above the melting point during a build. Furthermore, it is possible that further nucleation also occurs during the annealing process. Finally, the model does not demonstrate a decrease in bulk spherulite size with feed rate, as observed in the experimental data. This discrepancy is discussed further in the next section. 


\section{Discussion}

The thermal annealing of parts printed using PLA reveals a nonuniform semicrystalline structure that is templated into the part from the temperature and flow history during printing. Since thermal annealing is a proposed mechanism for increasing strength (16) and variation in spherulite size due to this templating effect is expected to affect mechanical properties (43), it is important to understand where and how these inhomogeneities arise.

In particular, we find that the crystalline microstructure varies spatially in the annealed wall, with smaller spherulites present near the weld between two printed filaments. This is captured, qualitatively and quantitatively, by a theoretical model that incorporates polymer melt rheology, heat transfer, and crystallization kinetics. Thus, our work highlights the critical need for materials characterization, in-line measurements, and physics-based models to adequately understand the relationship between printing conditions and properties in polymer-based additive manufacturing.

Inducing smaller spherulites in the weld region by controlling FIC could be exploited as a method to improve the mechanical properties of printed parts. In particular, decreasing spherulite size is found to lead to more ductile fracture (43). Furthermore, flow-enhanced crystallization can lead to a higher crystalline fraction $(44,45)$, thus it may also be possible to exploit FIC to increase the number of tie chains across filament-filament interfaces and consequently improve weld strength. Future work will be to measure weld strength as a function of FIC to test this hypothesis.

Similar FIC behavior also occurs in MatEx-printed PCL (24), with smaller spherulites forming near to the surface of a singly deposited filament, while the bulk of the filament is governed by slower quiescent kinetics. In contrast to ref (24), in this work, a discrepancy arises predicting the bulk crystal morphology found in the middle of a PLA filament comprising a multifilament wall. In particular, the bulk spherulite size increases with print speed, in contrast to the experimental observations. This discrepancy arises from neglecting to capture additional (asymmetric) heating and cooling cycles that occur during the creation of a multiple-filament wall.

For instance, it is evident in Figure 4 that the temperature of the weld line, as measured by infrared imaging, increases slightly to a peak prior to the exponential decay to the ambient temperature. This early-time temperature variation is absent in the temperature measurements of a single PCL filament, discussed in ref (24), and is not currently captured by the axisymmetric temperature model employed here. We propose that by combining the initial jump to the average temperature, as employed in this work, followed by a small degree of heating prior to the exponential decay region, then both the bulk crystal morphology, as well as the flow-enhanced weld region morphology, will be predictable. 
In particular, in the proposed temperature model (Eq 24), $T_{0}$ is chosen to ensure that the temperature at $t=0$ is equivalent to the average $\left(T_{N}+T_{a}\right) / 2$, as seen in one-dimensional modeling (10). Although this choice results in $t_{M}<\tau_{R}$ so that surface FIC occurs under all tested print conditions, as suggested by the experimental data, the resulting fitting parameter $A$ increases with print speed; equivalently, the cooling rate $1 / A$ decreases with print speed. Since smaller cooling rates produce fewer nuclei (Figure 12), larger spherulites grow at faster print speeds for this modeling approach. A second choice is to fit $T_{0}$ to the infrared imaging data. This results in a cooling rate $1 / A$ that increases with print speed and consequently spherulite size decreasing with print speed, in agreement with the experiment. Yet, for these temperature parameters, a flow-enhanced boundary layer is not created for all printing conditions since $t_{M}>\tau_{R}$.

Clearly, in order to predict both the bulk and the weld morphology, further modeling efforts are required to accurately capture the early-time temperature evolution induced by deposition of multiple layers during the creation of a wall. Quantitative predictions also rely on knowledge of the quiescent nucleation rate at low temperatures, which is extremely difficult to measure. Furthermore, the model as developed can predict structural differences in three dimensions, although we have only focused on the cross section of the printed parts in this work. Additional structural and orientational characterization of the printed part in the flow direction can be performed using optical microscopy or microbeam Xray scattering techniques and is the subject of future work.

\section{Conclusion}

In this chapter, we have combined material characterization and in-line crystallization measurements with a nonisothermal, molecularly aware, flowenhanced crystallization model to demonstrate how MatEX flow can affect the crystal morphology of a PLA printed part. In particular, the model demonstrates that residual stretch induced by the deposition process enhances nucleation at the surface of a deposited filament, while the bulk of the filament is governed by slower quiescent kinetics. Thus, more nuclei and a greater degree of space filling is achieved along the outer edge, compared to the middle of a deposited filament; an effect that is unapparent in the experimental measurements post-printing. However, since the crystal fraction remains small at the free surface, it is unlikely to affect interdiffusion dynamics or mechanical strength at the filament-filament welds (24), at least prior to annealing.

Thermal annealing is a proposed method for increasing the strength of printed parts by increasing the crystal fraction $(18,46)$. However, full comprehension of the mechanical properties requires understanding how the resulting morphology (i.e., spherulite size) varies across a single filament. In particular, nonuniform crystal morphology will lead to variations in the elastic moduli $(47,48)$. In this 
chapter, optical microscopy of the thermally annealed wall reveals a nonuniform spherulite size that emerges from the cross-sectional variation in nucleation density predicted by the model. In particular, smaller spherulites are formed in the weld regions between deposited filaments due to FIC, whereas spherulites at the center of the filaments are larger. We have demonstrated that only a singlefilament model is required to accurately capture the flow-enhanced weld regions found in an annealed PLA MatEx-printed wall.

We propose that FIC in the weld region may be employed as a method to improve the strength of MatEx-printed parts. In particular, better space filling near the interface is suggested to increase the availability of spherulites to form tie chains across the weld line (24). Furthermore, inducing smaller spherulites in the weld region may also lead to more ductile fracture (43). However, further experiments measuring weld strength as a function of FIC are required to explore this hypothesis.

\section{Acknowledgments}

The authors would like to acknowledge S. Orski at NIST for the GPC measurement and analysis used in the chapter. C. McIlroy would also like to thank R. Graham, University of Nottingham for fruitful discussions.

\section{References}

1. Turner, B. N.; Strong, R.; Gold, S. A. A Review of Melt Extrusion Additive Manufacturing Processes: I. Process Design and Modeling. Rapid Prototyping J. 2014, 20, 192-204.

2. Heller, B. P.; Smith, D. E.; Jack, D. A. Planar Deposition Flow Modeling of Fiber Filled Composites in Large Area Additive Manufacturing. Addit. Manuf. 2019, 25, 227-238.

3. Serdeczny, M. P.; Comminal, R.; Pedersen, D. B.; Spangenberg, J. Experimental Validation of a Numerical Model for the Strand Shape in Material Extrusion Additive Manufacturing. Addit. Manuf. 2018, 24, 145153.

4. Xia, H.; Lu, J.; Dabiri, S.; Tryggvason, G. Fully Resolved Numerical Simulations of Fused Deposition Modeling. Part I: Fluid Flow. Rapid Prototyping J. 2018, 24 (2), 463-476.

5. Gleadall, A.; Ashcroft, I.; Segal, J. VOLCO: A Predictive Model for 3D Printed Microarchitecture. Addit. Manuf. 2018, 21, 605-618. 
6. Mackay, M. E.; Swain, Z. R.; Banbury, C. R.; Phan, D. D.; Edwards, D. A. The performance of the hot end in a plasticating 3D printer. Journal of Rheology, 2017, 61 (2), 229-236.

7. Sun, Q.; Rizvi, G. M.; Bellehumeur, C. T.; Gu, P. Effect of Processing Conditions on the Bonding Quality of FDM Polymer Filaments. Rapid Prototyping J. 2008, 14, 72-80.

8. Song, Y.; Li, Y.; Song, W.; Yee, K.; Lee, K-Y.; Tagarielli, V. L. Measurements of the Mechanical Response of Unidirectional 3D-Printed PLA. Mater. Des. 2017, 123, 154-164.

9. Ge, T.; Pierce, F.; Perahia, D.; Grest, G. S.; Robbins, M. O. Molecular Dynamics Simulations of polymer welding: Strength from Interfacial Entanglements. Phys. Rev. Lett. 2013, 110, 098301.

10. McIlroy, C.; Olmsted, P. D. Disentanglement Effects on Welding Behaviour of Polymer Melts during the Fused-Filament-Fabrication Method for Additive Manufacturing. Polymer 2017, 123, 376-391.

11. Seppala, J. E.; Migler, K. D. Infrared Thermography of Welding Zones Produced by Polymer Extrusion Additive Manufacturing. Addit. Manuf. 2016, 12, 71-76.

12. D'Amico, A.; Peterson, A. M. An Adaptable FEA Simulation of Material Extrusion Additive Manufacturing Heat Transfer in 3D. Addit. Manuf. 2018, 21, 422-430.

13. Seppala, J. E.; Hoon Han, S.; Hillgartner, K. E.; Davis, C. S.; Migler, K. B. Weld Formation during Material Extrusion Additive Manufacturing. Soft Matter 2017, 13, 6761-6769.

14. Du, J.; Wei, Z.; Wang, X.; Wang, J.; Chen, Z. An Improved Fused Deposition Modeling Process for Forming Large-Size Thin-Walled Parts. J. Mater. Process. Technol. 2016, 234, 332-341.

15. Jarrousse, G. Self Adhesion of Semi-Crystalline Polymers between Their Glass Transition Temperature and Their Melting Temperature. Ph.D. Thesis, Université Pierre et Marie Curie-Paris VI, Paris, 2004.

16. Torres, J.; Cotelo, J.; Karl, J.; Gordon, A. P. Mechanical Property Optimization of FDM PLA in Shear with Multiple Objectives. JOM 2015, 67 (5), 1183-1193.

17. Chacón, J. M.; Caminero, M. A.; García-Plaza, E.; Núñez, P. J. Additive Manufacturing of PLA Structures Using Fused Deposition Modeling: Effect of process parameters on Mechanical Properties and Their Optimal Selection. Mater. Des. 2017, 124, 143-157.

18. Wang, L.; Gramlich, W. M.; Gardner, D. J. Improving the Impact Strength of Poly(lactic acid) (PLA) in Fused Layer Modeling (FLM). Polymer 2017, 114, 242-248.

19. Wittbrodt, B.; Pearce, J. M. The effects of PLA color on Material Properties of 3-D Printed Components. Addit. Manuf. 2015, 8, 110-116. 
20. McIlroy, C.; Olmsted, P. D. Deformation of an Amorphous Polymer during the Fused-Filament-Fabrication Method for Additive Manufacturing. $J$. Rheol. 2017, 61, 379-397.

21. Steenbakkers, R. J. A.; Peters, G. W. M. A Stretch-Based Model for FlowEnhanced Nucleation of Polymer Melts. J. Rheol. 2011, 55, 401-433.

22. Graham, R. S.; Olmsted, P. D. Coarse-Grained Simulations of Flow-Induced Nucleation in Semicrystalline Polymers. Phys. Rev. Lett. 2009, 103, 115702.

23. Northcutt, L. A.; Orski, S. V.; Migler, K. B.; Kotula, A. P. Effect of Processing Conditions on Crystallization Kinetics during Materials Extrusion Additive Manufacturing. Polymer 2018, 154, 182-187.

24. McIlroy C.; Graham, R. S. Modelling Flow-Enhanced Crystallization during the Fused-Filament-Fabrication of Semi-Crystalline Polymer Melts. Addit. Manuf. 2018, 24, 323-340.

25. Certain commercial equipment, instruments, or materials are identified in this chapter in order to specify the experimental procedure adequately. Such identification is not intended to imply recommendation or endorsement by the National Institute of Standards and Technology, nor is it intended to imply that the materials or equipment identified are necessarily the best available for the purpose.

26. Kotula, A. P.; Meyer, M. W.; De Vito, F.; Plog, J.; Hight Walker, A. R.; Migler, K. B. The Rheo-Raman Microscope: Simultaneous Chemical, Conformational, Mechanical, and Microstructural Measures of Soft Materials. Rev. Sci. Instrum. 2016, 87 (10), 105105.

27. Samuel, A. Z.; Zhou, M.; Ando, M.; Mueller, R.; Liebert, T.; Heinze, T.; Hamaguchi, H. O. Determination of Percent Crystallinity of Side-Chain Crystallized Alkylated-Dextran Derivatives with Raman Spectroscopy and Multivariate Curve Resolution. Anal. Chem. 2016, 88 (9), 4644-4650.

28. Jaumot, J.; Gargallo, R.; de Juan, A.; Tauler, R. A Graphical User-Friendly Interface for MCR-ALS: A New Tool for Multivariate Curve Resolution in MATLAB. Chemom. Intell. Lab. Syst. 2005, 76 (1), 101-110.

29. Likhtman, A. E.; Graham, R. S. Simple Constitutive Equation for Linear Polymer Melts Derived from Molecular Theory: Rolie-Poly Equation. $J$. Non-Newtonian Fluid Mech. 2003, 114 (1), 1-12.

30. Doi, M.; Edwards, S. F. The Theory of Polymer Dynamics; Oxford University Press: Oxford, 1988; Vol. 73.

31. Williams, M. L.; Landel, R. F.; Ferry, J. D. The Temperature Dependence of Relaxation Mechanisms in Amorphous Polymers and Other Glass-Forming Liquids. J. Am. Chem. Soc. 1955, 77 (14), 3701-3707.

32. Graham, R. S. Molecular Modelling of Entangled Polymer Fluids Under Flow. Ph.D. Thesis, University of Leeds, Leeds, U.K., 2002.

33. Peters, G. W.; Balzano, L.; Steenbakkers, R. J. Flow-Induced Crystallization. In Handbook of Polymer Crystallization; John Wiley \& Sons: Hoboken, NJ, 2013; pp 399-431. 
34. Avrami, M. Kinetics of Phase Change. I General Theory. J. Chem. Phys. 1939, 7 (12), 1103-1112.

35. Likhtman, A. E.; McLeish, T. C. Quantitative Theory for Linear Dynamics of Linear Entangled Polymers. Macromolecules 2002, 35 (16), 6332-6343.

36. https://reptate.readthedocs.io/ 21/02/2019

37. Lauritzen J. I., Jr.; Hoffman, J. D. Extension of Theory of Growth of ChainFolded Polymer Crystals to Large Undercoolings. J. Appl. Phys. 1973, 44 (10), 4340-4352.

38. Drieskens, M.; Peeters, R.; Mullens, J.; Franco, D.; Lemstra, P. J.; HristovaBogaerds, D. G. Structure Versus Properties Relationship of Poly(lactic acid). I. Effect of Crystallinity on Barrier Properties. J. Polym. Sci. Part B: Polym. Phys. 2009, 47 (22), 2247-2258.

39. Qin, D.; Kean, R. T. Crystallinity Determination of Polylactide by FT-Raman Spectrometry. Appl. Spectrosc. 1998, 52 (4), 488-495.

40. Binsbergen, F. L. Natural and Artificial Heterogeneous Nucleation in Polymer Crystallization. J. Polym. Sci.: Polym. Symp. 1977, 59 (1), 11-29.

41. Comminal, R.; Serdeczny, M. P.; Pedersen, D. B.; Spangenberg, J. Numerical Modeling of the Strand Deposition Flow in Extrusion-Based Additive Manufacturing. Addit. Manuf. 2018, 20, 68-76.

42. Kousiatza, C.; Karalekas, D. In-Situ Monitoring of Strain and Temperature Distributions during Fused Deposition Modeling Process. Mater. Des. 2016, 97, 400-406.

43. Way, J. L.; Atkinson, J. R.; Nutting, J. The Effect of Spherulite Size on the Fracture Morphology of Polypropylene. J. Mater. Sci. 1974, 9 (2), 293-299.

44. Chellamuthu, M.; Arora, D.; Winter, H. H.; Rothstein, J. P. Extensional Flow-Induced Crystallization of Isotactic Poly-1-Butene Using a Filament Stretching Rheometer. J. Rheol. 2011, 55 (4), 901-920.

45. White, E. E. B.; Winter, H. H.; Rothstein, J. P. Extensional-Flow-Induced Crystallization of Isotactic Polypropylene. Rheol. Acta 2012, 51 (4), 303314.

46. Srithep, Y.; Nealy, P.; Turng, L.-S. (2013). Effects of Annealing Time and Temperature on the Crystallinity and Heat Resistance Behavior of InjectionMolded Poly(lactic acid). Polym. Eng. Sci. 2013, 53 (3), 580-588.

47. Halpin, J. C.; Kardos, J. L. Moduli of Crystalline Polymers Employing Composite Theory. J. Appl. Phys. 1972, 43 (5), 2235-2241.

48. Humbert, S.; Lame, O.; Séguéla, R.; Vigier, G. A Re-Examination of the Elastic Modulus Dependence On Crystallinity In Semi-Crystalline Polymers. Polymer 2011, 52 (21), 4899-4909.

49. Cooper-White, J. J.; Mackay, M. E. Rheological Properties of Poly(lactides). Effect of Molecular Weight and Temperature on the Viscoelasticity of Poly(1lactic acid). J. Polym. Sci. Part B: Polym. Phys. 1999, 3 (15), 1803-1814.

50. Polylactic Acid (PLA, Polylactide). https://www.makeitfrom.com/materialproperties/Polylactic-Acid-PLA-Polylactide 21/02/2019 
